\title{
Distinctive signatures of space-time diffeomorphism breaking in EFT of inflation
}

\author{
Nicola Bartolo ${ }^{* 1,2}$, Dario Cannone ${ }^{\dagger 1,2}$, Angelo Ricciardone ${ }^{\ddagger 3}$, and Gianmassimo Tasinato ${ }^{\S 4}$ \\ ${ }^{1}$ Dipartimento di Fisica e Astronomia "G. Galilei", \\ Università degli Studi di Padova, via Marzolo 8, I-35131, Padova, Italy \\ ${ }^{2}$ INFN, Sezione di Padova, via Marzolo 8, I-35131, Padova, Italy \\ ${ }^{3}$ Faculty of Science and Technology, University of Stavanger, 4036, Stavanger, Norway \\ ${ }^{4}$ Department of Physics, Swansea University, Swansea, SA2 8PP, U.K.
}

November 24, 2015

\begin{abstract}
The effective field theory of inflation is a powerful tool for obtaining model independent predictions common to large classes of inflationary models. It requires only information about the symmetries broken during the inflationary era, and on the number and nature of fields that drive inflation. In this paper, we consider the case for scenarios that simultaneously break time reparameterization and spatial diffeomorphisms during inflation. We examine how to analyse such systems using an effective field theory approach, and we discuss several observational consequences for the statistics of scalar and tensor modes. For example, examining the three point functions, we show that this symmetry breaking pattern can lead to an enhanced amplitude for the squeezed bispectra, and to a distinctive angular dependence between their three wavevectors. We also discuss how our results indicate prospects for constraining the level of spatial diffeomorphism breaking during inflation.
\end{abstract}

\section{Introduction}

The most precise cosmological observations at our disposal [1, 2] are compatible with simple scenarios of inflation based on a single field model, that lead to an almost scale invariant, gaussian spectrum of scalar adiabatic perturbations. On the other hand, both the wealth of more precise data that will become available in the next few years, and the fact that some anomalies seem to be present in current observations (see [3] and, e.g., 4 4 for a review) suggest to keep an eye open to different possibilities.

The effective field theory of inflation (EFTI) is a powerful tool for obtaining model independent predictions for large classes of inflationary scenarios. It requires only information about the symmetries broken during the inflationary era, and on the number and nature of fields that drive inflation. The advantage of EFTI is that one has not to commit on specific realizations in order to deduce observable consequences. One writes the most general set of operators that satisfies the symmetry requirements and connects the coefficients of such operators with observable quantities. The EFTI has been successfully applied to cosmological models of single-field inflation, that break time reparameterization.

\footnotetext{
*nicola.bartolo@pd.infn.it

${ }^{\dagger}$ dario.cannone@pd.infn.it

${ }^{\ddagger}$ angelo.ricciardone@uis.no

$\S$ g.tasinato@swansea.ac.uk
} 
Space-time diffeomorphisms correspond to the invariance of the theory under the gauge symmetry of General Relativity:

$$
x^{\mu} \rightarrow x^{\prime \mu}\left(x^{\nu}\right),
$$

for arbitrary functions $x^{\prime \mu}\left(x^{\nu}\right)$ of the coordinates. During inflation, time-reparameterisation invariance

$$
t \rightarrow t+\xi\left(x^{\mu}\right)
$$

is broken. This is due to the existence of an 'inflationary clock' that breaks time diffeomorphisms and controls how much time is left before inflation ends. In models of single field inflation, there is a unique clock and only adiabatic modes can be generated on superhorizon scales. What is controlling the clock dynamics is what sources inflation, and at the same time causes the spontaneous breaking of de Sitter symmetry during the inflationary era. Studying the system at high energies, we can expect that gravity decouples. Time reparameterisation becomes a global symmetry, and its breaking gives rise to a massless Goldstone boson $\pi$. Its high energy dynamics faithfully describes the dynamics of the fluctuations of the inflationary clock. This thanks to Goldstone boson equivalence theorems, originally proven in quantum field theory for gauge symmetries [5], and more recently applied to the EFTI in [6, 7].

While time reparameterization invariance is certainly spontaneously broken by the source of inflation, it is interesting to explore the possibility that space diffeomorphisms are also broken during the inflationary epoch. After all, we are ignorant about what was really happening at the high energy scales and early times characterising inflation. In this paper, we consider cases where also the symmetry

$$
x^{i} \rightarrow x^{i}\left(t, x^{j}\right)
$$

is violated during inflation. This can be realised if there are fields that acquire a vev depending on spatial coordinates, as scalars $\phi=\phi\left(x^{i}\right)$, or alternatively if there are fields that select a preferred direction, as vector configurations that break rotational invariance. Concrete realisations of both these possibilities can be found, for example, in models where inflationary fields acquire vacuum expectation values along space-like directions, motivated by Solid Inflation [8, 9, 10, or inflationary set-ups involving vector fields (see e.g. [11, 12]).

A general EFT approach allows us to study in a model independent way the consequences of this particular symmetry breaking. The phenomenology of these models can be quite different with respect to standard scenarios. They can lead to a blue spectrum of gravity waves, anisotropic features in non-Gaussianities, and new couplings among different sectors (scalar-tensor-vector) of fluctuations (see e.g. 13]). Also, at the level of the background, scenarios that break space diffeomorphisms can accommodate models that break isotropy, possibly related to some of the anomalies in the CMB (see e.g. the recent review [4]). Hence, it is interesting to develop a model independent approach for studying the breaking of space diffeomorphisms during inflation, exploiting techniques based on the EFTI. This is the scope of this work. Preliminary studies have been made in [14, 15] (see also [16, 17. and the recent [18). Here we further develop this subject, directly working with a Goldstone action for fluctuations. When working at sufficiently high energies, we can expect that gravity decouples, and spatial diffeomorphisms reduce to global space translations and rotations: the breaking of these symmetries lead again to Goldstone bosons. In particular, a scalar 'phonon' appears, that we call $\sigma$ and that is associated with the broken translational invariance. This Goldstone field $\sigma$ interacts with the Goldstone boson $\pi$ associated with the breaking of time translations. Such couplings are constrained by non-linearly realised symmetries. They lead to interesting effects, that we analyse for the first time and that - as we will explain - are not obtained in the standard EFTI (i.e. where only time-reparameterisation invariance is broken) or Solid Inflation scenarios.

Indeed, we determine two broad physical effects that are distinctive of our set-up, that we analyze in detail:

- The first is specific of the scalar sector, and exploits the new couplings between the two scalar Goldstone modes of broken symmetries. We find potentially large contributions to inflationary observables, that can give sizeable effects even in the limit of small breaking of space diffeomorphisms. Such contributions lead to a change in the amplitude of the power spectrum of scalar 
fluctuations, and, more interestingly, direction dependent contributions to the squeezed limit of the scalar and tensor bispectra (in the sense that the bispectra depend non trivially on the angle between the three wavevectors, and can be parametrized with Legendre polynomials $P_{\mathrm{L}}$ and amplitude coefficients $c_{\mathrm{L}}$ as in [19]).

We discuss the physical consequences of these findings, pointing our similarities and differences with previous results in the literature, as Solid Inflation [8], inflationary models involving vector [20, 21] or higher spin [22] field components.

- The second effect is instead more specific of tensor sector, and exploits novel possibilities for tensors to couple with themselves and with scalars. Such possibilities are associated with operators that are allowed only if we break also space reparameterisation invariance during inflation. They can lead to a blue spectrum for gravitational waves, and moreover to a particular structure for the squeezed limit of tensor-scalar-scalar bispectra, that violates single field consistency relations and can lead to distinctive observable signatures.

We discuss these findings in Sections 2,5, and we conclude in Section 6, In the three Appendixes we give technical details about the decoupling limit and the strong coupling scale in our model and we estimate the size of a galaxy survey in order to give informations on the pattern of symmetries of our model.

\section{System under consideration}

General Relativity is built in terms of a powerful symmetry principle, diffeomorphism invariance:

$$
x^{\mu} \rightarrow x^{\mu}+\xi^{\mu}\left(x^{\nu}\right) .
$$

Such symmetry can be spontaneously broken by sources contributing to the energy momentum tensor that explicitly depend on the coordinates. For example, an homogeneous scalar field $\phi(t)$ breaks time reparameterization invariance, since its value is generally not preserved under the transformation $\phi\left(t+\xi^{0}\left(x^{\mu}\right)\right) \neq \phi(t)$. In this paper, we study the case of a system that spontaneously breaks all diffeomorphisms during inflation. The spontaneous breaking of diffeomorphisms implies that perturbations of symmetry-breaking fields transform non-linearly under diffeomorphisms. Such situation can be achieved if fields select preferred spatial directions, or depend explicitly on space-like coordinates.

The study of this system can be carried on following different approaches, that we now briefly discuss. The first approach consists on working in what is called the 'unitary gauge'. One makes the hypothesis that the system breaks diffeomorphism symmetries in such a way that a gauge can be selected, where the fluctuations of the fields sourcing inflation can be set to zero, and perturbations are stored in the metric only 1 . This gauge choice makes the counting of the degrees of freedom particularly simple, and provides a geometrical interpretation of the dynamical fluctuations. The possibility of making this gauge choice requires that we can work with at most four fields, that acquire vacuum expectation values spontaneously breaking the symmetry. We label them as $\phi^{\mu}, \mu=0, . ., 3$. We then assume that their own perturbations can be set to zero appropriately selecting the four functions $\xi^{\mu}$ in eq (4). This is our definition of unitary gauge (a similar condition was studied in [8]). In this gauge, the dynamical degrees of freedom are stored in the metric: the usual transverse, plus all the longitudinal polarisations of the graviton. The resulting theory can be seen as an effective theory of (Lorentz violating) massive gravity in a cosmological space-time [23, 24. Besides the two transverse helicities, the longitudinal graviton polarisations can account for at most four more degrees of freedom: two form a transverse vector and two are scalars. Notice that the scalars can both have healthy dynamics around a cosmological space-time (i.e. one of them does not necessarily correspond to a ghost, as in flat space [25]).

\footnotetext{
${ }^{1}$ Let us emphasise that this condition is not automatically satisfied in all models of inflation. Consider a system of twofields inflation, $\phi_{i}$ with $i=1,2$, where both fields contribute to inflation acquiring a time-dependent vev. Their perturbations transform non-trivially under time reparameterization, $\delta \phi_{i} \rightarrow \delta \phi_{i}+\partial_{t} \phi_{i} \xi^{0}$. Having a unique function $\xi^{0}$ to play with, we don't have enough freedom for setting both $\delta \phi_{i}$ to zero.
} 
While the unitary gauge is well suited for geometrically understanding the dynamical degrees of freedom, in our work we adopt a second approach to study an inflationary system with broken spacetime diffeomorphisms. We interpret the new dynamical modes that arise as Goldstone bosons of broken space-time symmetries. In order to do so, it is convenient to define our coordinates to be aligned with the background values of the fields that spontaneously break diffeomorphisms.

The vacuum expectation values for the symmetry breaking fields are

$$
\bar{\phi}^{0}=t, \quad \bar{\phi}^{i}=\alpha x^{i},
$$

$\bar{\phi}^{0}$ and $\bar{\phi}^{i}$ are respectively clock and rulers during inflation. The parameter $\alpha$ controls the breaking of spatial diffeomorphisms: we assume it to be small, and we will use it as an expansion parameter. Using the Stückelberg trick, we can restore full diffeomorphism invariance by introducing a set of four fields, $\pi$ and $\sigma^{i}$, and write the gauge invariant combinations $\phi^{\mu}$ as

$$
\phi^{0}=t+\pi, \quad \phi^{i}=\alpha x^{i}+\alpha \sigma^{i} .
$$

The Stückelberg fields $\pi$ and $\sigma^{i}$ transform under diffeomorphisms such to render the previous combinations gauge invariant. For the system that we consider, $\sigma_{i}$ can be decomposed into longitudinal $\sigma_{L}$ and transverse components $\sigma_{i}^{T}$. The longitudinal component $\sigma_{L}$ interacts with $\pi$, starting already at quadratic level: the interaction among these scalars will be the main topic of our work.

We make further assumptions: we would like to preserve homogeneity and isotropy, imposing extra internal symmetries on the field configuration [8],

$$
\phi^{i} \rightarrow O_{j}^{i} \phi^{j}, \quad \phi^{i} \rightarrow \phi^{i}+c^{i},
$$

where $O_{j}^{i} \in S O(3)$. We further assume an approximate shift symmetry $\phi^{0} \rightarrow \phi^{0}+c^{0}$, which is a technically natural assumption to protect the small time dependence of the coefficients that will appear in the action. Notice that these internal symmetries we impose act on field space. Diffeomorphism invariance of eq (1) acts on coordinate space instead, and is spontaneously broken in our system. Notice that our pattern of symmetry breaking is different from the recent [18, that breaks rotational symmetry in the internal field space.

With this in mind, we can write - at lowest order in a derivative expansion - the diffeomorphisms invariant action describing our system

$$
S=\int \mathrm{d}^{4} x \sqrt{-g}\left[\frac{1}{2} M_{\mathrm{Pl}}^{2} R+F\left(X, Y^{i}, Z^{i j}\right)\right]
$$

where $F$ is an arbitrary function, respecting the internal group of spacetime shifts and rotations (7) and $g$ is the determinant of the metric tensor. The building blocks that appear in the function $F$ are the operators:

$$
\begin{aligned}
X & =\partial_{\mu} \phi^{0} \partial_{\nu} \phi^{0} g^{\mu \nu}, \\
Y^{i} & =\partial_{\mu} \phi^{0} \partial_{\nu} \phi^{i} g^{\mu \nu}, \\
Z^{i j} & =\partial_{\mu} \phi^{i} \partial_{\nu} \phi^{j} g^{\mu \nu},
\end{aligned}
$$

where $i=1,2,3$. In what follows, we discuss the consequences of this form of the action for the dynamics of the Stückelberg fields.

\section{Inflationary background and fluctuation dynamics}

\subsection{The equations for the background}

Our first task is to determine the background evolution. We selected the background values for the fields that break diffeomorphisms to be aligned with the space-time coordinates, as in eq. (5). Such background fields are expected to drive inflation. We now consider what conditions our function $F$ have 
to satisfy, in order to generate a quasi-de Sitter period of inflationary expansion. We start assuming Friedmann-Robertson-Walker ansatz for the metric

$$
g_{\mu \nu}=\operatorname{diag}\left(-1, a^{2}, a^{2}, a^{2}\right)
$$

where $a$ is the scale factor of the universe. The energy-momentum tensor of our theory reads:

$$
\begin{aligned}
T_{\mu \nu} & =-\frac{2}{\sqrt{-g}} \frac{\delta S}{\delta g^{\mu \nu}}= \\
& =g_{\mu \nu} F-2\left(F_{X} \partial_{\mu} \phi^{0} \partial_{\nu} \phi^{0}+F_{Y^{i}} \partial_{\mu} \phi^{0} \partial_{\nu} \phi^{i}+F_{Z^{i j}} \partial_{\mu} \phi^{i} \partial_{\nu} \phi^{j}\right),
\end{aligned}
$$

where the subscripts of $F$ stand for the partial derivatives with respect to the operators $(9)$. When computed on the background values of the fields, eq (5), Einstein equations lead to the Friedmann equations (where $H=\dot{a} / a$ and overlines denote quantities evaluated on the background):

$$
\begin{aligned}
3 M_{\mathrm{Pl}}^{2} H^{2} & =\left(-\bar{F}-2 \bar{F}_{X}\right), \\
-2 M_{\mathrm{Pl}}^{2} \dot{H} & =-2\left(\bar{F}_{X}+\frac{\alpha^{2}}{a^{2}} \bar{F}_{Z}\right) .
\end{aligned}
$$

On the background, the operators (9) read

$$
\bar{X}=-1, \quad \bar{Y}^{i}=0, \quad \bar{Z}^{i j}=\frac{\alpha^{2} \delta^{i j}}{a^{2}(t)} .
$$

Notice that $Z^{i j}$ depends on $\alpha$ - being associated with the breaking of space diffeomorphisms - but it also explicitly depends on time, through the scale factor.

The isotropy of the background requires

$$
\bar{F}_{Z^{i j}}=\bar{F}_{Z} \delta_{i j}, \quad \bar{F}_{Y^{i}}=0 .
$$

Our configuration solves all the background equations of motion (included the ones associated with the fields $X, Y^{i}, Z^{i j}$ ) if the following condition is satisfied ${ }^{2}$

$$
2 \alpha^{2} \bar{F}_{X Z}=a^{2} F_{X}
$$

Using this information, we can express the slow-roll epsilon parameter $\epsilon=-\dot{H} / H^{2}$ as

$$
\epsilon=\frac{3 \bar{X} \bar{F}_{X}-\bar{Z} \bar{F}_{Z}}{-\bar{F}+2 \bar{X} \bar{F}_{X}}
$$

To obtain a phase of inflation we require $\epsilon \ll 1$, which, barring accidental cancellations, can be naturally obtained if the function $F$ has only a weak dependence on both $X$ and $Z$ :

$$
\left(\frac{\mathrm{d} \log F}{\mathrm{~d} \log X}, \frac{\mathrm{d} \log F}{\mathrm{~d} \log Z}\right) \ll 1 .
$$

Physically, the slow-roll parameter $\epsilon$ is associated with the 'ticks' of the inflationary clock. A small $\epsilon$ is associated with a configuration characterised by extremely slow ticks of the clock, corresponding to a quasi-de Sitter space-time. The rhythm of the inflationary clock ticks also varies, and is controlled by a second, independent slow-roll parameter $\eta=\dot{\epsilon} /(\epsilon H)$. A small $\eta$ ensures that changes in the rate of the inflationary clock occur slowly, so to provide a sufficiently long period of inflation. The condition $|\eta| \ll 1$ requires

$$
\left|\eta=2 \epsilon+\frac{6 \bar{F}_{X Z}+2 \bar{Z} \bar{F}_{Z}+2 \bar{Z}^{2} \bar{F}_{Z^{2}}}{-3 \bar{F}_{X}-\bar{Z} \bar{F}_{Z}}\right| \ll 1 .
$$

\footnotetext{
${ }^{2}$ Notice that this equation is equivalent to the continuity equation. In the limit $\alpha \rightarrow 0$, one consistently obtain a limit $F_{X} \rightarrow 0$, which is the limit of the continuity equation for a $F(X)$ theory when the symmetry $\phi_{0} \rightarrow \phi_{0}+c$ is taken as an exact symmetry.
} 
Having a small value for $\epsilon$ implies, at leading order in slow-roll, that the quantities

$$
\frac{F_{X}}{F} \quad \text { and } \quad \frac{F_{Z}}{a^{2} F},
$$

are constant that do not depend on space-time coordinates (notice the explicit presence of the scale factor in the second one).

Our set-up does not correspond to a single clock model of inflation. We can identify two independent contributions that control the inflationary clock. The first is associated with the breaking of time reparameterisation, through an explicitly time-dependent background value for the field $\bar{\phi}^{0}$, as in eq (5). The second is related with the time dependence of the quantity $\bar{Z}^{i j}$ introduced in eq (14). $\bar{Z}^{i j}$ is associated with the breaking of space diffeomorphisms, and is defined in terms of the inflationary rulers $\bar{\phi}^{i}$ in eq (5). $\bar{Z}^{i j}$ acquires a dependence on the scale factor $a(t)$ (due to the contraction with the spatial part of the metric). A similar fact is found also in Solid Inflation 8 .

These two contributions to the energy momentum tensor both independently control the inflationary clock. Hence we are not dealing with a purely adiabatic system. And indeed, we will see next that we can identify two dynamical scalar fluctuations around our background configuration, each corresponding to a Goldstone boson of a different broken symmetry. The non-adiabatic properties of our set-up are quite distinctive though, and are the topic of the remaining discussion.

\subsection{Quadratic action for Stückelberg fields}

We now discuss the structure of quadratic fluctuations of the transverse components of the metric, and the Stückelberg fields $\pi, \sigma^{i}$ introduced in eq (6) as

$$
\phi^{0}=t+\pi, \quad \phi^{i}=\alpha\left(x^{i}+\sigma^{i}\right),
$$

as fields restoring diffeomorphism invariance.

In principle, besides the (self-)interactions of $\pi$ and $\sigma^{i}$, also interactions of these fields with the metric components $\delta g^{00}, \delta g^{0 i}, \delta g^{i j}$ should be taken into account. However, we can consider the theory at very short distances - corresponding to energy scales $E=k / a \gg H$ - where the effects of gravity backreaction can be neglected. Gravitational modes decouple: the local diffeomorphisms of general relativity reduce to the global symmetries of Lorentz boosts and translations. In this decoupling limit the fields $\pi$ and $\sigma^{i}$ can be interpreted as Goldstone bosons of these broken global symmetries, and these degrees of freedom interact only with themselves ${ }^{3}$

After these considerations, let us then focus on the system in a high energy decoupling limit, where the Stückelberg fields can be identified with Goldstone bosons of broken space-time diffeomorphisms. We start writing the quadratic actions for these systems $4^{4}$ at leading order in slow-roll parameters and the parameter $\alpha$, neglecting gravitational corrections in our decoupling limit:

$$
\begin{aligned}
S^{(S)=} & \int \mathrm{d}^{4} x a^{3}\left[\left(-\bar{F}_{X}+2 \bar{F}_{X^{2}}\right) \dot{\pi}^{2}+\left(\bar{F}_{X}+\frac{\alpha^{2} \bar{F}_{Y^{2}}}{2 a^{2}}\right) \frac{\partial_{i} \pi \partial^{i} \pi}{a^{2}}+\alpha^{2}\left(\frac{\bar{F}_{Y^{2}}}{2}-\bar{F}_{Z}\right) \dot{\sigma}_{L}^{2}\right. \\
& \left.+\alpha^{2}\left(\bar{F}_{Z}+\alpha^{2} \frac{2 \bar{F}_{Z Z}}{a^{2}}+\alpha^{2} \frac{2 \bar{F}_{Z^{2}}}{a^{2}}\right) \frac{\partial_{i} \sigma_{L} \partial^{i} \sigma_{L}}{a^{2}}+\alpha^{2} \frac{4 \bar{F}_{X Z}}{a^{2}} \sqrt{-\nabla^{2}} \dot{\pi} \sigma_{L}-\alpha^{2} \frac{\bar{F}_{Y^{2}}}{a^{2}} \sqrt{-\nabla^{2}} \pi \dot{\sigma}_{L}\right], \\
S^{(V)=} & \int \mathrm{d}^{4} x a^{3}\left[\left(\frac{\bar{F}_{Y^{2}}}{2}-\bar{F}_{Z}\right) \dot{\sigma}_{T}^{i} \dot{\sigma}_{T, i}+\left(\bar{F}_{Z}+2 \frac{\bar{F}_{Z Z}}{a^{2}}\right) \frac{\partial_{j} \sigma_{T}^{i} \partial^{j} \sigma_{T, i}}{a^{2}}\right], \\
S^{(T)=} & \int \mathrm{d}^{4} x a^{3} \frac{1}{8}\left[M_{\mathrm{Pl}}^{2}\left(\dot{\gamma}_{i j} \dot{\gamma}^{i j}-\frac{\partial_{k} \gamma_{i j} \partial^{k} \gamma^{i j}}{a^{2}}\right)+\alpha^{2}\left(\frac{\bar{F}_{Z}}{a^{2}}+\frac{\alpha^{2} \bar{F}_{Z Z}}{2 a^{4}}\right) \gamma_{i j} \gamma^{i j}\right] .
\end{aligned}
$$

\footnotetext{
${ }^{3}$ See Appendix A for a technical discussion of decoupling limit in our set-up.

${ }^{4}$ The internal symmetries (7) limit the possible operators that can appear in the action. For example, deriving $F$ twice with respect to $Z^{i j}$ gives $\mathrm{d} F / \mathrm{d} Z^{i j} \mathrm{~d} Z^{k l}=F_{Z Z} \delta_{i k} \delta_{j l}+F_{Z^{2}} \delta_{i j} \delta_{k l}$.
} 
where $S, V, T$ represent the scalar, vector and tensor sectors respectively. The fields $\pi$ has dimension of inverse of mass, and $\sigma$ is dimensionless. The field $\sigma^{i}$ has been decomposed in a (vector) transverse component and a (scalar) longitudinal one:

$$
\sigma^{i}=\sigma_{T}^{i}+\frac{\partial^{i} \sigma_{L}}{\sqrt{-\nabla^{2}}} .
$$

As explained above, when all diffeomorphisms are broken, in general six degrees of freedom are dynamical: two scalar fluctuations, the two components of a transverse vector, and the two helicities of a traceless transverse tensor.

The most evident consequence of our set-up is that we now have a system of two interacting scalars, $\pi$ and $\sigma$, Goldstone bosons of two different symmetries. These scalars are coupled through distinctive derivative operators, controlled by the pattern of symmetry breaking in our system. Notice that masses, and non-derivative couplings among the fields, do not arise at our level of approximation, because of the symmetries (7) and since we are neglecting gravitational effects. Our action is different with respect to the EFTI action for multifield inflation [26]. While the previous actions are quadratic in perturbations, also non-linear interactions can be included: we will do so in Section 5.

We should also check that the actions $22,(23)$ and $(24)$ do not lead to dangerous instabilities. For example, the coefficient of the time kinetic operators should have the right sign:

$$
\begin{aligned}
-\bar{F}_{X}+2 \bar{F}_{X}^{2} & >0, \\
\bar{F}_{Y^{2}}-2 \bar{F}_{Z} & >0 .
\end{aligned}
$$

At the same time one should impose that the speeds of sound,

$$
\begin{aligned}
c_{\pi}^{2} & =\frac{\bar{F}_{X}+\bar{\alpha}^{2} F_{Y^{2}} / 2 a^{2}}{\bar{F}_{X}-2 \bar{F}_{X^{2}}}, \\
c_{\sigma}^{2} & =\frac{\bar{F}_{Z}+2 \alpha^{2} \bar{F}_{Z Z} / a^{2}+2 \alpha^{2} \bar{F}_{Z^{2}} / a^{2}}{F_{Z}-F_{Y^{2}} / 2}, \\
c_{T}^{2} & =\frac{\bar{F}_{Z}+2 \alpha^{2} \bar{F}_{Z Z} / a^{2}}{\bar{F}_{Z}+\bar{F}_{Y^{2}} / 2},
\end{aligned}
$$

lie in the interval $0<c_{s}^{2} \leq 1$, where $s \equiv \pi, \sigma, T$. The complete list of relations between the coefficients that one can derive is not particularly illuminating, but we checked that there are regions of the parameter space where there are no dangerous instabilities. The allowed range of parameters will of course be important when trying to compare with cosmological observations, but this topic goes beyond the scope of the present work. Moreover, to avoid excessive time evolution for these quantities during inflation, we can impose that the "slow-roll" parameter associated with the speeds of sound should be small:

$$
s_{c}=\frac{\dot{c}_{s}}{c_{s} H} \ll 1
$$

This again can give constraints on combinations of parameters in the action, when comparing with observations. Moreover it suggests that, like $\bar{F}_{X}$ and $\bar{F}_{Z} / a^{2}$, also the other coefficients in the action, like for example $\bar{F}_{Y^{2}} / a^{2}$ are slowly varying and can be taken as constant. It would be interesting to see what are the consequences of relaxing this assumption and consider non-trivial time dependencies.

\subsection{The expression for the curvature perturbation}

There are two commonly used gauge invariant definitions of curvature perturbations, the curvature perturbation on uniform density hypersurfaces, $\zeta$, and the comoving curvature perturbation $\mathcal{R}$. In single field inflation, on superhorizon scales these quantities are conserved, and coincide up to a sign: see e.g. 27] for a review.

This implies that any result obtained in the aforementioned sub-horizon, decoupling limit remains valid also at superhorizon scales, since the curvature perturbation gets frozen there in single-field 
inflation. As we explained above, our system is strictly speaking not single field, and non-adiabatic contributions can arise. They are controlled by a small quantity though - the parameter $\alpha$ that characterises the breaking of space diffeomorphisms, see eq (5). The expression for the comoving curvature perturbation in the decoupling limit reads for our system ${ }^{5}$

$$
\mathcal{R}=\frac{H}{\left(-M_{\mathrm{Pl}}^{2} \dot{H}\right)}\left[\left(-\bar{F}_{X}+\frac{\alpha^{2} \bar{F}_{Y^{2}}}{2 a^{2}}\right) \pi+\alpha^{2}\left(2 \bar{F}_{Z}-\bar{F}_{Y^{2}}\right) \frac{\dot{\sigma}_{L}}{\sqrt{-\nabla^{2}}}\right] .
$$

In the limit of $\alpha$ small, this expression reduces to the 'single-field' expression

$$
\mathcal{R}=-H \pi
$$

commonly used in EFTI [6] (where $\mathcal{R}$ is dubbed $\zeta$ ). In what follows, we will work in a small $\alpha$ limit, so that the definition (33) is sufficiently accurate, and we can neglect its time-dependence at superhorizon scales. This is also justified because, nevertheless, we will find interesting potentially sizeable corrections to the $n$-point functions for $\mathcal{R}$ associated with the complete breaking of diffeomorphism invariance.

\section{The two-point functions}

\subsection{The power spectrum for scalar fluctuations}

In this section we consider the consequences of the new symmetry pattern in the second order action of the scalar perturbations. First, let us rewrite the action $(22)$ in terms of the normalized fields $\hat{\pi}$ and $\hat{\sigma}$

$$
\begin{gathered}
\hat{\pi}=\sqrt{2\left(-\bar{F}_{X}+2 \bar{F}_{X^{2}}\right)} \pi, \quad \hat{\sigma}=\alpha \sqrt{2\left(\frac{\bar{F}_{Y^{2}}}{2 a^{2}}-\frac{\bar{F}_{Z}}{a^{2}}\right)} \sigma_{L} \\
S^{(S)}=\int \mathrm{d}^{4} x a^{3}\left[\frac{1}{2}\left(\dot{\hat{\pi}}^{2}-c_{\pi}^{2} \frac{\partial_{i} \hat{\pi} \partial^{i} \hat{\pi}}{a^{2}}\right)+\frac{1}{2} a^{2}\left(\dot{\hat{\sigma}}^{2}-c_{\sigma}^{2} \frac{\partial_{i} \hat{\sigma} \partial^{i} \hat{\sigma}}{a^{2}}\right)+\alpha \lambda_{1} \sqrt{-\nabla^{2}} \dot{\hat{\pi}} \hat{\sigma}+\alpha \lambda_{2} \sqrt{-\nabla^{2}} \hat{\pi} \dot{\hat{\sigma}}\right]
\end{gathered}
$$

where the speeds of sound are written in eqs. 28, 29 and

$$
\begin{aligned}
\lambda_{1} & =\frac{2 \bar{F}_{X Z} / a^{2}}{\sqrt{\left(-\bar{F}_{X}+2 \bar{F}_{X^{2}}\right)\left(\bar{F}_{Y^{2}} / 2 a^{2}-\bar{F}_{Z} / a^{2}\right)}}, \\
\lambda_{2} & =\frac{-\bar{F}_{Y^{2}} / a^{2}}{2 \sqrt{\left(-\bar{F}_{X}+2 \bar{F}_{X^{2}}\right)\left(\bar{F}_{Y^{2}} / 2 a^{2}-\bar{F}_{Z} / a^{2}\right)}} .
\end{aligned}
$$

The normalization of the fields have been defined so that the parameters $\lambda_{1}, \lambda_{2}$ are constant at leading order in slow-roll. The price to pay is that we leave an explicit factor of $a^{2}$ in front of the 'kinetic action' for the Goldstone mode $\sigma$ in eq (35).

We can see that, for small values of the parameter $\alpha$, the interaction terms that mix the two fields can be treated as perturbations on top of a free Lagrangian for the two scalars involved. Thanks to this fact, we can perturbatively compute the spectrum for the fluctuation $\pi$ by the following procedure. First, we evaluate it at zero order in the parameter $\alpha$. Then, we compute perturbative corrections in $\alpha$, using the in-in formalism. This calculation will provide a quantitative way to evaluate how the second Goldstone boson $\sigma$ affects the properties of the two-point function of $\pi$ and the curvature perturbation. Physically, we are interested to this question because we have learned that the contribution to curvature perturbation $\mathcal{R}$ is mostly due the field $\pi$, in the limit of small values for $\alpha$ (see section $(3.3)$ ). On the other hand we will learn that contributions of $\sigma$ to two and higher point functions of $\mathcal{R}$ can be sizeable even in the limit of small $\alpha$.

\footnotetext{
${ }^{5}$ In the flat gauge the comoving curvature perturbation is defined as $\mathcal{R}=H \delta u$, where $\delta u$ is the longitudinal component of the perturbed 4-velocity of the fluid [28].
} 
Figure 1: Leading diagram for computing the symmetry breaking contributions to $\left\langle\pi^{2}\right\rangle$.

Let us then proceed computing the power spectrum for $\pi$. The zeroth order power spectrum is straightforward to obtain:

$$
\left\langle\hat{\pi}_{\vec{k}_{1}} \hat{\pi}_{\vec{k}_{2}}\right\rangle=(2 \pi)^{3} \delta\left(\vec{k}_{1}+\vec{k}_{2}\right) \frac{2 \pi^{2}}{k_{1}^{3}} \hat{\mathcal{P}}_{0},
$$

where

$$
\hat{\mathcal{P}}_{0}=\frac{H^{2}}{4 \pi^{2} c_{\pi}^{3}} .
$$

Using the normalization coefficient (34), the power spectrum of the original fields $\pi$ reads:

$$
\hat{\mathcal{P}}_{0}=\frac{H^{2}}{8 \pi^{2} c_{\pi}^{3}\left(-\bar{F}_{X}+2 \bar{F}_{X^{2}}\right)}=\frac{H^{2}}{8 \pi^{2} c_{\pi}\left(-\bar{F}_{X}-\alpha^{2} \bar{F}_{Y^{2}} / 2 a^{2}\right)},
$$

where we used the definition of the speed of sound (28). Taking $\alpha \ll 1$ and using eq. (13), this result reduces to the standard result of single-field inflation with only time-diffeomorphism breaking, as in that case $\bar{F}_{X}=M_{\mathrm{Pl}}^{2} \dot{H}$ :

$$
\hat{\mathcal{P}}_{0}(\alpha \ll 1)=\frac{H^{4}}{8 \pi^{2} c_{\pi}\left(-\bar{F}_{X}\right)}=\frac{H^{2}}{8 \pi^{2} M_{\mathrm{Pl}}^{2} \epsilon c_{\pi}} .
$$

The effect of the interaction terms in the second order action (35) can be now computed using the in-in formalism [33, 34. The leading correction to the power spectrum is given by

$$
\delta\left\langle\hat{\pi}_{\vec{k}_{1}} \hat{\pi}_{\vec{k}_{2}}(\tau)\right\rangle=-\int_{\tau_{\min }}^{\tau} d \tau_{1} \int_{\tau_{\min }}^{\tau_{1}} d \tau_{2}\left\langle\left[\left[\hat{\pi}_{\vec{k}_{1}}^{(0)} \hat{\pi}_{\vec{k}_{2}}^{(0)}(\tau), \mathcal{H}_{\mathrm{int}}^{(2)}\left(\tau_{1}\right)\right], \mathcal{H}_{\mathrm{int}}^{(2)}\left(\tau_{2}\right)\right]\right\rangle,
$$

where $\mathcal{H}_{\text {int }}^{(2)}$ is the second order interaction hamiltonian and $\tau_{\min }$ corresponds to the time at which the contribution of the long mode starts to become dominant. This correction is represented as mass insertion diagram in Fig 1. In our case, from (35), we have:

$$
\mathcal{H}_{\mathrm{int}}^{(2)}(\tau)=\mathcal{H}_{\mathrm{int}, 1}^{(2)}(\tau)+\mathcal{H}_{\mathrm{int}, 2}^{(2)}(\tau)
$$

where

$$
\begin{aligned}
& \mathcal{H}_{\text {int }, 1}^{(2)}(\tau)=\frac{\alpha \lambda_{1}}{(H \tau)^{3}} \int \frac{d^{3} k}{(2 \pi)^{3}}|k| \hat{\sigma}_{\vec{k}}^{(0)}(\tau) \hat{\pi}_{-\vec{k}}^{\prime(0)}(\tau), \\
& \mathcal{H}_{\text {int }, 2}^{(2)}(\tau)=\frac{\alpha \lambda_{2}}{(H \tau)^{3}} \int \frac{d^{3} k}{(2 \pi)^{3}}|k| \hat{\pi}_{\vec{k}}^{(0)}(\tau) \hat{\sigma}_{-\vec{k}}^{\prime(0)}(\tau) .
\end{aligned}
$$

The field operators can be expanded in terms of their Fourier modes

$$
\begin{aligned}
& \hat{\pi}_{\vec{k}}=u_{k} a_{\vec{k}}+u_{k}^{*} a_{-\vec{k}}^{\dagger}, \\
& \hat{\sigma}_{\vec{k}}=v_{k} b_{\vec{k}}+v_{k}^{*} b_{-\vec{k}}^{\dagger},
\end{aligned}
$$

where the creation and annihilation operators respect the commutation rules:

$$
\left[a_{\vec{k}}, a_{-\vec{k}^{\prime}}^{\dagger}\right]=(2 \pi)^{3} \delta^{(3)}\left(\vec{k}+\vec{k}^{\prime}\right), \quad\left[b_{\vec{k}}, b_{-\vec{k}^{\prime}}^{\dagger}\right]=(2 \pi)^{3} \delta^{(3)}\left(\vec{k}+\vec{k}^{\prime}\right), \quad\left[a_{\vec{k}}, b_{-\vec{k}^{\prime}}^{\dagger}\right]=0 .
$$

This is because the eigenfunctions for the two scalar modes are the solution of the classical equations of motion, derived from the (free) action that can be read from (22). For the field $\pi$ we have:

$$
u_{k}^{\prime \prime}-\frac{2}{\tau} u_{k}^{\prime}+c_{\pi}^{2} k^{2} u_{k}=0
$$


where we have used $a H=-1 / \tau+\mathcal{O}(\epsilon)$. This has the standard solution (after choosing the BunchDavies vacuum and fixing the integration constants):

$$
u_{\vec{k}}(\tau)=-\frac{H}{\sqrt{2 c_{\pi}^{3} k^{3}}}\left(1+i k c_{\pi} \tau\right) e^{-i k c_{\pi} \tau} .
$$

In the same way one can write the equation of motion for the field $\sigma$ :

$$
v_{k}^{\prime \prime}-\frac{4}{\tau} v_{k}^{\prime}+c_{\sigma}^{2} k^{2} v_{k}=0,
$$

whose solution is:

$$
v_{k}(\tau)=-\frac{H^{2}}{\sqrt{2 c_{\sigma}^{5} k^{5}}}\left(-3-3 i k c_{\sigma} \tau+c_{\sigma}^{2} k^{2} \tau^{2}\right) e^{-i k c_{\sigma} \tau} .
$$

Notice that the vacuum wave configuration for the field $\sigma$, (51), is different with respect to the vacuum configuration for $\pi$, eq (49). The difference is due to the presence of the scale factor $a^{2}$ in front of the kinetic term for $\sigma$, in the quadratic action for fluctuations.

Performing the commutators and plugging the interaction hamiltonian 44 into in 42 , we arrive to integrals like:

$$
\begin{aligned}
\delta\left\langle\hat{\pi}_{\vec{k}_{1}} \hat{\pi}_{\vec{k}_{2}}(\tau)\right\rangle= & -\frac{4 \alpha^{2} \lambda_{1}^{2}}{H^{6}} \operatorname{Re}\left[\int_{\tau_{\min }}^{\tau} \frac{\mathrm{d} \tau_{1}}{\tau_{1}^{3}} \int_{\tau_{\min }}^{\tau_{1}} \frac{\mathrm{d} \tau_{2}}{\tau_{2}^{3}} \mathrm{k}^{2} \times\right. \\
& \left.\left(v_{k_{1}}\left(\tau_{2}\right) v_{k_{1}}^{*}\left(\tau_{1}\right) u_{k_{1}}^{\prime}\left(\tau_{2}\right) u_{k_{1}}^{*}(\tau)\left(u_{k_{1}}^{\prime}\left(\tau_{1}\right) u_{k_{1}}^{*}(\tau)-\text { c.c }\right)\right)\right] .
\end{aligned}
$$

Together with this, there are also the integrals coming from the substitution of $\mathcal{H}_{\text {int,2 }}$ (45), proportional to $\lambda_{2}^{2}$ and the mixed contributions proportional to $\lambda_{1} \lambda_{2}$. These integrals are dominated by the contributions at the times when the modes are outside the horizon, as on sub-horizon scale the oscillatory phases in the mode functions suppress the result ${ }^{6}$. Setting $c_{\sigma} \simeq c_{\pi}$ for simplicity, we can take $\tau_{\min }=-1 / c_{\pi} k$ and perform the integral analitically. We find the main contribution in the large scale limit:

$$
\frac{\delta \mathcal{P}}{\mathcal{P}_{0}}=\frac{3 \alpha^{2} \lambda_{1} N_{k}\left(3 \lambda_{2}-\lambda_{1}\left(3 N_{k}+6 \gamma_{E}+11-\log (64)\right)\right.}{c_{\pi}^{2}},
$$

where $\gamma_{E}$ is the Euler gamma and $N_{k}$ is the number of efolds from the time when the mode $k$ exits the horizon until the end of inflation. Notice that in eq (52) the integrand contains a factor of $k^{2}$, associated with the derivative interactions among the modes $\pi$ and $\sigma$. This factor compensates for the non-standard form of the vacuum solution (51) for $\sigma$, proportional to $k^{-5 / 2}$ at small scales (and not to $k^{-3 / 2}$ as it usually happens), leading to a scale invariant correction to the power spectrum.

One can see that even when the breaking of spatial diffeomorphisms is small, $\alpha \ll 1$, the effect of the interaction between the field $\sigma$ and the field $\pi$ can still be sizeable, as it is enhanced by the e-fold number $N_{k}$. In the limit of large $N_{k}$, the dominant correction to the power spectrum scales as

$$
\frac{\delta \mathcal{P}}{\mathcal{P}_{0}}=-\frac{9 \alpha^{2} \lambda_{1}^{2} N_{k}^{2}}{c_{\pi}^{2}} .
$$

This quantity can be non-negligible even if $\alpha$ is small, since the product $\alpha N_{k}$ can be sizeable (say of order one) being it enhanced by $N_{k}$. The logarithmic enhancement of the power spectrum has a similar behavior already met in other set-ups, see e.g. [35] or the review [36 7]. These are novel effects that we first point out in this paper, and are essentially due to the interplay between our two Goldstone bosons during inflation. They are physical, and arise even if the breaking of space diffeomorphisms is small, since they are enhanced by the e-fold number.

\footnotetext{
${ }^{6}$ The spurious divergences in the UV disappear when slightly deforming the countour of the time integration in the imaginary direction: see 33 .

${ }^{7}$ Notice that, since we are not in single field inflation, these enhancement effects cannot be 'gauged away' by a rescaling of the curvature perturbation. See e.g. [37] or the reviews [39].
} 
Since for small $\alpha$ the curvature perturbation $\mathcal{R}$ is proportional to $\pi$ through the simple relation (33), we can write the following modified expression to the power spectrum for $\mathcal{R}$, induced by a logenhancement due to the Goldstone boson $\sigma$ :

$$
\mathcal{P}_{\mathcal{R}} \simeq \frac{H^{2}}{8 \pi^{2} M_{\mathrm{Pl}}^{2} \epsilon c_{\pi}}\left(1-\frac{9 \alpha^{2} \lambda_{1}^{2} N_{k}^{2}}{c_{\pi}^{2}}\right) .
$$

Although for the case of scalar power spectrum the correction induced by our pattern for breaking spatial diffeomorphisms amounts to a change in the amplitude, for higher point functions we can have more relevant, direction dependent effects, as we will discuss in Section 5

\subsection{The power spectrum for tensor fluctuations}

The effect of breaking spatial diffeomorphisms can have interesting effects also in the tensor sector. This fact has been already explored in [14, 15. At the level of two-point functions involving tensor modes, the main difference with the standard case is associated with the possibility of assigning a non-vanishing mass to the tensors, since a mass operator is allowed by the absence of diffeomorphism invariance. The resulting set-up can then be considered as an effective theory of (Lorentz-violating) massive gravity during inflation. It would be interesting to find a consistent, UV complete theory of massive gravity that allows us to have a large graviton mass during cosmological inflation, and a small graviton mass after inflation ends. Nevertheless, in our approach based on EFT we do not need to rely on the existence of any specific UV realisation, and simply work with the most general set of operators, order by order in a field expansion.

Normalizing the tensor field as $\gamma_{i j}=\sqrt{2} \hat{\gamma}_{i j} / M_{\mathrm{Pl}}$ the quadratic Lagrangian for the two polarization modes of the gravitational fields has the form:

$$
\mathcal{L}=\frac{1}{4} \sqrt{-g}\left[\partial_{\mu} \hat{\gamma}_{i j} \partial^{\mu} \hat{\gamma}^{i j}-m^{2} \hat{\gamma}_{i j} \hat{\gamma}^{i j}\right]
$$

where $m^{2}=\alpha^{2}\left(\bar{F}_{Z}+\alpha^{2} \bar{F}_{Z Z} / 2 a^{2}\right) / M_{\mathrm{Pl}}^{2} a^{2}$.

To compute the power spectrum, one decomposes $h_{i j}$ into helicity modes,

$$
\hat{\gamma}_{i j}=\int \frac{\mathrm{d}^{3} k}{(2 \pi)^{3}} \sum_{s} \epsilon_{i j}^{(s)} \hat{\gamma}_{\vec{k}}^{(s)} \mathrm{e}^{\mathrm{i} \mathbf{k} \cdot \mathbf{x}},
$$

where $s= \pm$ is the helicity index and $\epsilon_{i j}$ is the polarization tensor. Making the redefinition $\hat{\gamma}_{\vec{k}}=h_{\vec{k}} / a$, the equation of motion (in conformal time $d \eta=d t / a(t)$ and neglecting slow-roll corrections) reads:

$$
h_{\vec{k}}^{\prime \prime}+\left[k^{2}-\frac{1}{\tau^{2}}\left(\nu^{2}-\frac{1}{4}\right)\right] h_{\vec{k}}=0,
$$

where $\nu=9 / 4-m^{2} / H^{2}$. The generic solution (for real $\nu$ ) is 8

$$
h_{\vec{k}}=\frac{\sqrt{\pi}}{2} \mathrm{e}^{\mathrm{i}(\nu+1 / 2) \frac{\pi}{2}} \sqrt{\eta} H_{\nu}^{(1)}(k \eta),
$$

where $H_{\nu}^{(1)}$ is the Hankel's function of the first kind. At this point one can easily find the tensor power spectrum

$$
\mathcal{P}_{T} \simeq \frac{2 H^{2}}{\pi^{2} M_{\mathrm{Pl}}^{2}}\left(\frac{k}{k *}\right)^{3-2 \nu}
$$

where:

$$
n_{T} \simeq m^{2} / H^{2}
$$

\footnotetext{
${ }^{8}$ For imaginary $\nu$ one can define a new $\tilde{\nu}=\mathrm{i} \nu$ and solve the differential equations in the same way. However in this case the power spectrum would be suppressed by the ration $H / m$ and fall rapidly on very large scales [40].
} 
Together with the standard $-2 \epsilon$ contribution to $n_{T}$, which can be easily found taking into account the slow-roll dependence in the equation of motion, this shows a non-trivial behaviour of the tensor tilt [14]:

$$
n_{T}=-2 \epsilon+\frac{2}{3} \frac{m^{2}}{H^{2}}
$$

As the $m^{2} / H^{2}$ contribution adds with a positive sign, if the mass of the tensor is large enough, then the spectrum could become blue [14, 16]. Moreover this would happen without inconsistencies, since it would not violate the Null Energy Condition, which is related to a change of sign of $\dot{H}$ [41. The interplay between the negative contribution of $\epsilon$ given by the time-diffeomophism breaking part and the positive contribution given by the breaking of space diffeomorphisms is a non-standard feature of this particular symmetry pattern. Notice also that, even though massive tensors are not constant after horizon exit, their evolution is very small as it is controlled by the small parameter $\alpha$. Indeed, if we take the limit $\alpha \ll 1$, tensor mass becomes completely negligible and we come back to the standard form of the tensor wave function:

$$
\gamma_{\vec{k}}=\frac{H}{M_{\mathrm{Pl}} k^{3 / 2}}(1+\mathrm{i} k \tau) \mathrm{e}^{-\mathrm{i} k \tau}
$$

So we find that breaking spatial diffeomorphisms provides qualitatively new effects in the power spectrum of tensor fluctuations. Other interesting effects arise when studying the bispectrum, as we are going to see in the next section.

\section{The three-point functions}

In this section, we examine non-linear, cubic interactions among cosmological fluctuations. In particular, we will study bispectra involving scalar and tensor fluctuations. The study of bispectra is conceptually important since their squeezed limits are very informative for what concern general features of the physics driving inflation. For example, it is known that in models with adiabatic fluctuations only, appropriate squeezed limits of three-point functions involving scalars and tensors, e.g. $\left\langle\mathcal{R}^{3}\right\rangle$ and $\left\langle\gamma \mathcal{R}^{2}\right\rangle$, are related to the tilt of the scalar power spectrum [33, 42,

When non-adiabatic interactions are turned on, these consistency relations are violated in a way that depends on the model one considers. We are interested in understanding general features of how the breaking of space-time diffeomorphisms affects the squeezed limits of three-point functions. We find that the breaking of such symmetry leads to (tunable) quadrupolar contributions to these quantities (corresponding to $c_{\mathrm{L}=2}$ contributions in the parameterisation of [19]) besides "pure" local (monopole $\left.c_{\mathrm{L}=0}\right)$ contributions in the squeezed limit.

Similar results have been already found in specific models, as Solid Inflation or models with vector fields [8, 9, 19, 20, but our EFT approach allows to generalize these results and understand them as due to a specific pattern of symmetry breaking.

As done in the case of the power spectrum, we are mostly interested on operators that are specifically associated with the simultaneous breaking of time and space reparameterization invariance, since these operators can lead to effects that have not been studied so far, when breaking separately time 6 and space 8 diffeomorphisms. Moreover, such effects can be sizeable, rendering them physically interesting even in a limit of small $\alpha$, the parameter associated with the breaking of spatial diffeomorphisms.

Given these motivations, the operators that we consider are specific of our construction that simultaneously break space and time diffeomorphisms. Up to second order in the parameter $\alpha$ they are the following:

$$
\begin{gathered}
\frac{8 \alpha^{2} \bar{F}_{X^{2} Z}}{3 a^{2}} \dot{\pi}^{2} \partial_{i} \sigma^{i} \\
\alpha^{2} \bar{F}_{Y^{2}}\left(\dot{\pi} \dot{\sigma}^{i} \dot{\sigma}_{i}-\frac{\dot{\pi} \dot{\sigma}^{i} \partial_{i} \pi}{a^{2}}+\frac{\gamma_{i j} \dot{\sigma}^{i} \partial^{j} \pi}{a^{2}}-\frac{\gamma_{i j} \partial^{i} \pi \partial^{j} \pi}{a^{4}}-\frac{\dot{\sigma}^{i} \partial_{j} \sigma_{i} \partial^{j} \pi}{a^{2}}+\frac{\partial_{j} \sigma_{i} \partial^{i} \pi \partial^{j} \pi}{a^{4}}\right), \\
\frac{2}{3} \alpha^{2} \bar{F}_{Y^{2} X}\left(-\dot{\pi} \dot{\sigma}^{i} \dot{\sigma}_{i}+\frac{2 \dot{\pi} \dot{\sigma}^{i} \partial_{i} \pi}{a^{2}}\right) .
\end{gathered}
$$




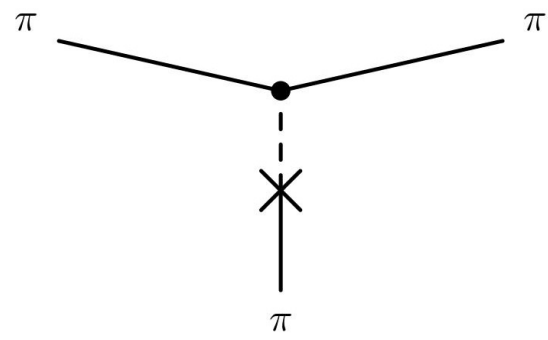

Figure 2: Leading diagram for computing the symmetry breaking contributions to $\left\langle\mathcal{R}^{3}\right\rangle$.

Here we are mostly interested in exploring interesting phenomenological consequences of our approach. On the other hand, the analysis of interactions can also be theoretically important to estimate the strong coupling scale at which unitarity bounds are violated in scattering experiments. We do not discuss this argument in the main text, but we develop it in Appendix B.

\subsection{The bispectrum for scalar fluctuations}

We start discussing how the operators breaking simultaneously space and time diffeomorphisms affect the squeezed limit of the curvature three-point function. The bispectrum of the curvature perturbation is defined as:

$$
\left\langle\mathcal{R}\left(\vec{k}_{1}\right) \mathcal{R}\left(\vec{k}_{2}\right) \mathcal{R}\left(\vec{k}_{3}\right)\right\rangle=(2 \pi)^{3} \delta^{3}\left(\vec{k}_{1}+\vec{k}_{2}+\vec{k}_{3}\right) \mathcal{B}\left(\vec{k}_{1}, \vec{k}_{2}, \vec{k}_{3}\right) .
$$

As for the two-point function, we compute the contributions of the symmetry breaking operators using a perturbative approach based on the in-in formalism. We take the quantity $\alpha$ controlling the breaking of space diffeomorphisms as a perturbation parameter. In the limit of small $\alpha$, the curvature perturbation is proportional to the Goldstone mode $\pi$, up to small corrections, and is conserved on superhorizon scales: see the discussion in Section 3.3, and the expression (33) for the curvature perturbations.

We then start with computing the contribution to the tree level bispectrum of the Goldstone $\pi$, due to the mixing with the Goldstone $\sigma$. We consider the contribution associated with the diagram represented in Fig. 2. In the limit of small $\alpha$, the operators that we consider are associated with a mass insertion second order hamiltonian $\mathcal{H}^{(2)}$, given by $(43)$, and by the third order operator

$$
\frac{\alpha}{\sqrt{2\left(\bar{F}_{X}+2 \bar{F}_{X^{2}}\right)}}\left[\left(\lambda_{2}+\lambda_{3}\right) \frac{\dot{\hat{\pi}} \partial^{i} \dot{\hat{\sigma}} \partial_{i} \hat{\pi}}{\sqrt{-\nabla^{2}}}-\lambda_{2} \frac{\partial_{j} \partial_{i} \hat{\sigma} \partial^{i} \hat{\pi} \partial^{j} \hat{\pi}}{a^{2} \sqrt{-\nabla^{2}}}-\lambda_{4} \dot{\hat{\pi}}^{2} \sqrt{-\nabla^{2}} \hat{\sigma}\right],
$$

that we express in terms of normalized fields (34). The new parameters $\lambda_{3}$ and $\lambda_{4}$ are defined as

$$
\begin{aligned}
& \lambda_{3}=\frac{4 \bar{F}_{Y^{2} X} / a^{2}}{3 \sqrt{\left(-\bar{F}_{X}+2 \bar{F}_{X}^{2}\right)\left(\bar{F}_{Y^{2}} / 2 a^{2}-\bar{F}_{Z} / a^{2}\right)}}, \\
& \lambda_{4}=\frac{8 \bar{F}_{X^{2} Z} / a^{2}}{3 \sqrt{\left(-\bar{F}_{X}+2 \bar{F}_{X}^{2}\right)\left(\bar{F}_{Y^{2}} / 2 a^{2}-\bar{F}_{Z} / a^{2}\right)}},
\end{aligned}
$$

while $\lambda_{1}$ and $\lambda_{2}$ are defined in (36), (37).

Then, the integral that we need to compute (see Fig 2 ) is

$$
\left\langle\hat{\pi}_{\vec{k}_{1}} \hat{\pi}_{\vec{k}_{2}} \hat{\pi}_{\vec{k}_{3}}\right\rangle=-\int_{\tau_{\min }}^{0} \mathrm{~d} \tau_{1} \int_{\tau_{\min }}^{\tau_{1}} \mathrm{~d} \tau_{2}\left\langle 0\left|\left[\mathcal{H}^{(3)}\left(\tau_{1}\right),\left[\mathcal{H}^{(2)}\left(\tau_{2}\right), \hat{\pi}_{k_{1}}(\tau) \hat{\pi}_{k_{2}}(\tau) \hat{\pi}_{k_{3}}(\tau)\right]\right]\right| 0\right\rangle .
$$

where the third-order interaction $\mathcal{H}^{(3)}$ can be extracted from (68). Let us make some example of the kind of integrals one has to study. Consider taking $\mathcal{H}^{(2)}$ as eq. (44) and $\mathcal{H}^{(3)}$ with only the operator 
$\partial_{j} \sigma_{i} \partial^{i} \pi \partial^{j} \pi$ from eq. (68). Then the form of the integral is:

$$
\begin{gathered}
\left\langle\hat{\pi}^{3}\right\rangle=2 \operatorname{Re}\left\{\alpha^{2} \frac{-\lambda_{1} \lambda_{2}}{\sqrt{2\left(-\bar{F}_{X}+2 \bar{F}_{X^{2}}\right)}} \int_{-\infty}^{0} \frac{\mathrm{d} \tau_{1}}{\left(-H \tau_{1}\right)^{2}} \int_{-\infty}^{\tau_{1}} \frac{\mathrm{d} \tau_{2}}{\left(-H \tau_{2}\right)^{3}} \delta^{3}\left(\vec{k}_{1}+\vec{k}_{2}+\vec{k}_{3}\right)\right. \\
\left(\vec{k}_{1} \cdot \vec{k}_{2}\right)\left(\vec{k}_{1} \cdot \vec{k}_{3}\right)\left[\lambda_{1} \hat{\sigma}_{k_{1}} \hat{\sigma}_{k_{1}}^{*} \hat{\pi}_{k_{1}}^{\prime} \hat{\pi}_{k_{1}}^{*}\left(\hat{\pi}_{k_{2}} \hat{\pi}_{k_{2}}^{*} \hat{\pi}_{k_{3}} \hat{\pi}_{k_{3}}^{*}-\text { c.c }\right)\right. \\
\left.\left.+\lambda_{2} \hat{\sigma}_{k_{1}}^{\prime} \hat{\sigma}_{k_{1}}^{*} \hat{\pi}_{k_{1}} \hat{\pi}_{k_{1}}^{*}\left(\hat{\pi}_{k_{2}} \hat{\pi}_{k_{2}}^{*} \hat{\pi}_{k_{3}} \hat{\pi}_{k_{3}}^{*}-\text { c.c }\right)\right]\right\} .
\end{gathered}
$$

All the other integrals we compute have a similar structure. To keep computations simple and analytical, we assume that the sound speeds are equal, $c_{\pi}=c_{\sigma}$. We recall that, when computing the power spectrum, we were finding log-enhanced contributions. We can expect the same amplification effects to occur here for the case of a squeezed bispectrum when a long wavelength mode $(k \rightarrow 0)$ is already outside the horizon. Then we can consider one of the momenta $k_{1}=k_{L} \rightarrow 0$, while the other two are assumed with equal lenght $k_{2} \sim k_{3}=k_{S}$, and evaluate the integral from the time when the mode exits the horizon $\tau=-1 / c_{\pi} k_{1}$ until the end of inflation. Summing all the terms arising from the operators 68 and focusing only on the leading contributions we obtain

$$
\left\langle\hat{\pi}_{\vec{k}_{1}} \hat{\pi}_{\vec{k}_{2}} \hat{\pi}_{\vec{k}_{3}}\right\rangle=\frac{\alpha^{2} H^{5}}{16 c_{\pi}^{10} k_{L}^{3} k_{S}^{3}} \frac{1}{\sqrt{2\left(-\bar{F}_{X}+2 \bar{F}_{X^{2}}\right)}}\left[9 c_{\pi}^{2} \lambda_{1} \lambda_{4}+\left(3 \lambda_{1}+\lambda_{2}\right)\left(\lambda_{2}+\lambda_{3}\right) c_{\pi}^{2} \hat{S}_{2}-27 \lambda_{1} \lambda_{2} \hat{S}_{1}\right] \log \left(\frac{k_{L}}{k_{S}}\right),
$$

where $\hat{S}_{1}, \hat{S}_{2}$ refer to scalar products between versors of momenta:

$$
\begin{aligned}
& \hat{S}_{1}=\left(\hat{k}_{1} \cdot \hat{k}_{2}\right)\left(\hat{k}_{1} \cdot \hat{k}_{3}\right)+\left(\hat{k}_{2} \cdot \hat{k}_{3}\right)\left(\hat{k}_{1} \cdot \hat{k}_{2}\right)+\left(\hat{k}_{1} \cdot \hat{k}_{3}\right)\left(\hat{k}_{2} \cdot \hat{k}_{3}\right), \\
& \hat{S}_{2}=\hat{k}_{1} \cdot \hat{k}_{2}+\hat{k}_{2} \cdot \hat{k}_{3}+\hat{k}_{1} \cdot \hat{k}_{3} .
\end{aligned}
$$

In the squeezed limit, they reduce to:

$$
\hat{S}_{1}=-\cos ^{2} \theta, \quad \hat{S}_{2}=1-2 \cos ^{2} \theta,
$$

where $\theta$ is the angle between the long and the short wavelengths modes. From the three-point functions for $\pi$, as discussed above, we can extract the three-point function for the curvature perturbation $\mathcal{R}$. Using the normalization (34) together with the Friedmann eq. 13 and the definition of the speed of sound (28), we can write the squeezed limit of the three-point function for curvature pertubation, up to second order in $\alpha$, as:

$$
\begin{aligned}
& \left\langle\mathcal{R}^{3}\right\rangle_{k_{L} \rightarrow 0} \simeq(2 \pi)^{3} \delta^{3}\left(\vec{k}_{1}+\vec{k}_{2}+\vec{k}_{3}\right) \alpha^{2} \frac{\mathcal{P}\left(k_{L}\right) \mathcal{P}\left(k_{S}\right)}{k_{L}^{3} k_{S}^{3}} \frac{\pi^{4}}{c_{\pi}^{4}} \times \\
& \times\left\{\left[9 c_{\pi}^{2} \lambda_{1} \lambda_{4}+\left(3 \lambda_{1}+\lambda_{2}\right)\left(\lambda_{2}+\lambda_{3}\right) c_{\pi}^{2}\right]+\left[27 \lambda_{1} \lambda_{2}-2\left(3 \lambda_{1}+\lambda_{2}\right)\left(\lambda_{2}+\lambda_{3}\right) c_{\pi}^{2}\right] \cos ^{2} \theta\right\} \log \left(\frac{k_{L}}{k_{S}}\right),
\end{aligned}
$$

where we only write the log-enhanced contributions to this quantity. Let us comment on the physical consequences of this result:

- Even if, in the squeezed limit, the curvature three-point function is suppressed by a factor of $\alpha^{2}$ (a parameter that we consider small) it is nevertheless enhanced by a factor $\log \left(k_{L} / k_{S}\right)$, a quantity that can be of order of the number of e-folds of inflation:

$$
\log \left(\frac{k_{L}}{k_{S}}\right) \simeq N_{k}
$$

This means that, as for the case of the power spectrum, we find a log-enhanced contribution. The same considerations of Section 4.1 hold here: since we have non-adiabatic fluctuations only, these effects are physical and cannot be gauged away with a redefinition of coordinates. Notice that, moreover, the three-point function is enhanced by a large power of the sound speed $\left(1 / c_{\pi}^{4}\right)$ that can also considerably increase its size, in the case that $c_{\pi}<1$. 
- Interestingly, we find a non-trivial angular dependence of the squeezed limit of the bispectrum. The squeezed bispectrum can be expressed as a sum of two contributions, a monopole plus a quadrupole, with tunable coefficients depending on the parameters $\lambda_{i}$. An angular dependent squeezed bispectrum has been also found in other works in the literature, as Solid Inflation [8, or inflation with vector fields [19, 20, 29, 30, 31, 32, or in models with higher spin fields [22. In those realizations, the coefficients in front of each contributions (monopole and quadrupole) are fixed by the model. In our set-up based on an EFT approach to inflation, we have been able to identify classes of operators that allow to obtain more general squeezed limits for the bispectrum, with arbitrary coefficients in front of each angular-dependent contribution. We can then identify a possible origin of these effects as due to particular patterns of space-time diffeomorphism breaking. It would be interesting to find concrete models that obtain our operators from a fundamental set-up.

\subsection{Tensor-scalar-scalar bispectra and consistency relations}

In this subsection we examine how breaking space-time diffeomorphisms affects the bispectra involving tensor and scalar fluctuations. Observables associated with three-point functions involving tensor modes are becoming particularly interesting, since they are sensitive to the behavior of gravity at the high scales of inflation, and since the future promises advances in observational efforts to detect primordial tensor modes. We start with a brief review on the present theoretical and observational status of our knowledge of tensor-scalar-scalar bispectra; then we pass to discuss new results we obtain within the EFT of inflation with broken space-time diffeomorphisms.

\subsubsection{Motivations}

In light of the amount of precise measurements that are becoming available, it is important to select the best observables that will clarify the physics responsible for driving inflation. Among the predictions of inflation there is one that affects both the CMB and the LSS of the universe: it is the correlation between primordial scalar and tensor perturbations (gravitational waves) 33.

This tensor-scalar-scalar (TSS) correlation, that is present in all the inflationary models, generates a local power quadrupole in the power spectrum of the scalar perturbations when the wavelength of the tensor mode is much bigger than the scalar one (squeezed limit), giving rise to an apparent local departure from statistical isotropy. This observable is a useful quantity to discriminate among the plethora of inflationary models. Moreover this long wavelength tensor mode leaves a precise imprints (dubbed fossils) on the observed mass distribution of the universe. The properties of the correlation functions are dictated by symmetries that have a crucial role in constraining the form of correlation functions, and the corresponding consistency relations and their violation $42,43,44,45,46,47,48,49$. 4.

In the next years we will see an increased dedicated effort in trying to detect gravitational waves [50, 51, 52, 53, 54, 55, 56.

In [57] it has been shown that, in the case of single-clock models, that are space-diffeomorphism invariant, a quadrupole contribution to the TSS is cancelled. In particular, a quadrupole contribution arises, proportional to the number of efolds, that is exactly compensated by late-time projection effects that leave a negligible amplitude for the power quadrupole. However, when the conditions of singleclock [40, invariance under space diffeomophism [8, 9, 58], slow-roll evolution [59, 60] are evaded, then the consistency relation is violated, the cancellation is not perfect and we get a possibly detectable amplitude for the local power quadrupole. In [61] it has been shown how the violations of the slow-roll dynamics in non-attractor inflation and of space-diffeomorphism invariance in Solid Inflation bring to the violation of the consistency relation in the TSS correlation function with a consequent enhancement in the local quadrupole. In the case of non-attractor inflation, the limits from CMB on the statistical isotropy [3, 62] constrain the effects on non-observable scales since the transition from the non-attractor phase to the attractor one is found to happen before the time when the current observable universe left the horizon during the inflationary phase. In the Solid Inflation model, instead, the violation of the consistency relation is related to the violation of the diffeomophism invariance and, more interestingly, the observable anisotropic effects are spread on much smaller scales and so potentially detectable in 
the next future galaxies surveys. In a recent paper [63] the effect of the violation of the consistency relation has been computed in the Quasi-Single-Field model: a two fields model where one of the two has a mass near the Hubble scale $H$. From the non-trivial four-point function they estimate the size of the galaxy survey necessary to detect the effect of the tensor-scalar-scalar consistency violation.

\subsubsection{New results using the EFTI for broken space-time diffeomorphisms}

Our model, violating the invariance under space diffeomorphism, leads to a violations of the consistency relation of the tensor-scalar-scalar correlator, as we are going to discuss.

Following [45] the three-point function, in the case of a tensor-scalar-scalar interaction, can be re-defined as

$$
\left\langle\gamma_{\vec{k}_{1}}^{s} \mathcal{R}_{\vec{k}_{2}} \mathcal{R}_{\vec{k}_{3}}\right\rangle \equiv(2 \pi)^{3} \delta^{3}\left(\vec{k}_{1}+\vec{k}_{2}+\vec{k}_{3}\right)\left\langle\gamma_{\vec{k}_{1}}^{s} \mathcal{R}_{\vec{k}_{2}} \mathcal{R}_{\vec{k}_{3}}\right\rangle^{\prime}
$$

where the primed correlator is related to the bispectrum by

$$
\left\langle\gamma_{\vec{k}_{1}}^{s} \mathcal{R}_{\vec{k}_{2}} \mathcal{R}_{\vec{k}_{3}}\right\rangle^{\prime} \equiv \epsilon_{i j}^{s} \hat{k}_{2}^{i} \hat{k}_{3}^{j} \mathcal{B}\left(k_{1}, k_{2}, k_{3}\right)
$$

and $\epsilon_{i j}^{s}$ is the polarization tensor of the tensor mode.

Considering the limit in which the momentum of the tensor $\left(k_{1}\right)$ is identified with $k_{L}$ (long wavelength) and the momenta of the scalars $\left(k_{2}, k_{3}\right)$ are identified with $k_{S}$ (short wavelengths), when the consistency relation for the tensor-scalar-scalar correlator is satisfied, the bispectrum can be expressed like

$$
\mathcal{B}\left(k_{L}, k_{S}, k_{S}\right) \equiv-\frac{1}{2} P_{\gamma}\left(k_{L}\right) P_{\mathcal{R}}\left(k_{S}\right) \frac{\partial \ln P_{\mathcal{R}}\left(k_{S}\right)}{\partial \ln k_{S}},
$$

that, in single-field slow-roll models, translates in a quantity proportional to $\left(n_{s}-4\right)$, where $n_{s}$ is the scalar spectral index, as calculated by Maldacena in 33 .

In our case from the third order action 65 we can read that the tensor-scalar-scalar bispectrum has two contributions, that we can schematically write as

$$
\mathcal{B}\left(k_{1}, k_{2}, k_{3}\right)=\mathcal{B}_{[\gamma \partial \pi \partial \pi]}\left(k_{1}, k_{2}, k_{3}\right)+\mathcal{B}_{\left[\gamma \partial \dot{\sigma}_{L} \partial \pi\right]}\left(k_{1}, k_{2}, k_{3}\right) .
$$

These two contributions are associated with our novel operators corresponding to the fourth and third terms in 65). They add to the other contributions already present in EFTI and Solid Inflation (that we do not consider here), and can be computed using the in-in formalism. The (normalized) scalar Fourier wavefunctions are defined in 46 while for the tensor perturbations we use

$$
\gamma_{i j, \vec{k}}=\sum_{s= \pm} \epsilon_{i j}^{s}(\hat{k})\left[c_{\vec{k}}^{s} \gamma_{k}+\left(c^{s}\right)_{-\vec{k}}^{\dagger} \gamma_{k}^{*}\right]
$$

where " $s$ " represents the two polarizations of the tensor and the creation and annihilation operators respect the following commutation relation

$$
\left[c_{\vec{k}}, c_{-\vec{k}^{\prime}}^{\dagger}\right]=(2 \pi)^{3} \delta^{(3)}\left(\vec{k}+\vec{k}^{\prime}\right) \delta_{s s^{\prime}} .
$$

The scalar wave functions for the two scalar goldstones are given by (49) and (51) while for the tensor we can take the standard expression $\sqrt{63}$, since $\alpha^{2}$-correction to the wave function would be subleading when considered in this interactions $\}^{9}$ that are already proportional to $\alpha^{2}$.

The effect of the long wavelenght tensor mode on the two scalars is encoded in the squezeed limit $\left(k_{L} \ll k_{S}\right)$ of the bispectrum $\left\langle\gamma_{k_{L}} \pi_{k_{S}} \pi_{k_{S}}\right\rangle$. The first contribution that we obtain can be computed at tree-level, following 33 .

$$
\left\langle\gamma_{k_{L}} \hat{\pi}_{k_{S}} \hat{\pi}_{k_{S}}\left(\tau_{0}\right)\right\rangle=-i \int_{\tau_{m i n}}^{\tau_{0}} d \tau\left\langle\left[\gamma_{\vec{k}_{L}}\left(\tau_{0}\right) \hat{\pi}_{\vec{k}_{S}}\left(\tau_{0}\right) \hat{\pi}_{\vec{k}_{S}}\left(\tau_{0}\right), \mathcal{H}_{\gamma \partial \pi \partial \pi}^{(3)}(\tau)\right]\right\rangle
$$

and it gives

\footnotetext{
${ }^{9}$ The effects of modified wavefunctions could be interesting, in principle, when considered in the other bispectra which are not proportional to the small $\alpha^{2}$, like e.g. the standard $1 / c_{s}^{2}$ bispectrum.
} 


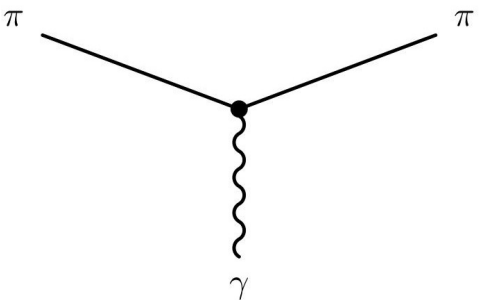

(a)

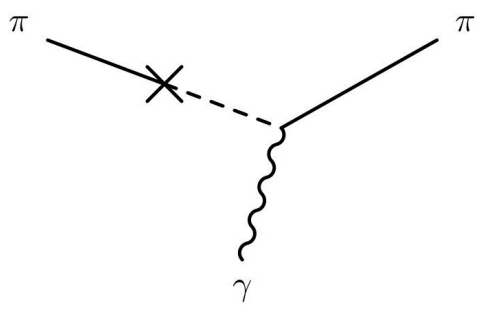

(b)

Figure 3: Leading consistency violating contributions to the TSS bispectrum $\langle\gamma \pi \pi\rangle$

$$
\mathcal{B}_{[\gamma \partial \mathcal{R} \partial \mathcal{R}]}\left(k_{L}, k_{S}, k_{S}\right)=\frac{H^{2}}{M_{P l}^{2}} \frac{\alpha^{2}}{\left(F_{X}-2 F_{X^{2}}\right)^{2}} \frac{F_{Y^{2}}}{a^{2}} \frac{3 H^{4}}{4 c_{\pi}^{5}}\left(\frac{1}{k_{S}^{3} k_{L}^{3}}\right) .
$$

Rewriting this contribution in terms of the curvature and tensor power spectra, we find a violation of the consistency relation in the tensor-scalar-scalar bispectrum. This since our result is proportional to the quantity $F_{Y}^{2}$ that is not related to the scalar spectral tilt; moreover it is not associated with the other observables met so far, so we do not have bounds on its size, although for naturalness reasons we do not expect it to be large. Let us also emphasize that such contribution to the TSS bispectrum is a distinctive feature of our set-up that simultaneously breaks time and space diffeomorphisms.

Interestingly, this is not the dominant contribution to the bispectrum: from the second interaction term in eq 82 we have

$$
\left\langle\gamma_{k_{L}} \hat{\pi}_{k_{2}} \hat{\pi}_{k_{3}}\right\rangle=-\int_{\tau_{m i n}}^{0} \mathrm{~d} \tau_{1} \int_{\tau_{m i n}}^{\tau_{1}} \mathrm{~d} \tau_{2}\left\langle\left[\mathcal{H}^{(3)}\left(\tau_{1}\right),\left[\mathcal{H}^{(2)}\left(\tau_{2}\right), \gamma_{k_{L}}(\tau) \hat{\pi}_{k_{2}}(\tau) \hat{\pi}_{k_{3}}(\tau)\right]\right]\right\rangle,
$$

where $\mathcal{H}^{(2)}$ is given by $(43)$ and

$$
\mathcal{H}_{[\gamma \partial \hat{\sigma} \partial \hat{\pi}]}^{(3)}=-\frac{2 \alpha}{M_{P l}} \lambda_{2} \int \mathrm{d}^{3} x a^{3} \frac{\gamma_{i j} \partial^{i} \hat{\sigma}^{\prime} \partial^{j} \hat{\pi}}{\sqrt{-\nabla^{2}}} .
$$

Performing the integral, considering the limit in which the tensor momentum $k_{L} \rightarrow 0$ is much smaller than the scalar momenta $k_{S}$, the result reads:

$$
\mathcal{B}_{[\gamma \partial \dot{\sigma} \partial \pi]}\left(k_{L}, k_{S}, k_{S}\right)=\frac{\alpha^{2} \lambda_{2} \lambda_{1}}{8 M_{P l}} \frac{H^{4}}{c_{\pi}^{5}}\left(\frac{1}{k_{S}^{3} k_{L}^{3}}\right) \log \left(\frac{2 c_{\pi} k_{S}}{k_{L}}\right) .
$$

This contribution is the dominant violating contribution to the three-point tensor-scalar-scalar correlation function. Indeed, although it is suppressed by a small parameter $\alpha$, it has a log-enhancement of the same kind we studied in the previous sections, that can be of the order of the number of e-folds.

Going back to the original correlator $\langle\gamma \pi \pi\rangle$, and considering only the leading contribution, rewriting it in terms of the curvature perturbation we find

$$
\mathcal{B}_{[\gamma \partial \mathcal{R} \partial \mathcal{R}]}\left(k_{L}, k_{S}, k_{S}\right) \supset \frac{\alpha^{2}}{M_{P l}^{2}} \frac{\lambda_{2} \lambda_{1}}{\left(-F_{X}+2 F_{X^{2}}\right)} \frac{H^{6}}{4 c_{\pi}^{5}}\left(\frac{1}{k_{S}^{3} k_{L}^{3}}\right) \log \left(\frac{2 c_{\pi} k_{S}}{k_{L}}\right) .
$$

or in terms of the scalar and tensor power spectra

$$
\mathcal{B}_{[\gamma \partial \mathcal{R} \partial \mathcal{R}]}\left(k_{L}, k_{S}, k_{S}\right) \supset P_{\gamma}\left(k_{L}\right) P_{\mathcal{R}}\left(k_{S}\right) \frac{\alpha^{2} \lambda_{1} \lambda_{2}}{4 c_{\pi}^{2}} \log \left(\frac{2 c_{\pi} k_{S}}{k_{L}}\right),
$$

and this our final result.

As anticipated, when a long wavelength tensor mode correlates with the density (scalar) fluctuations in the tensor-scalar-scalar squeezed bispectrum a local power quadrupole is generated. This contribution, that appears like a departure from statistical isotropy, shows an infrared-divergent behaviour, 
that becomes negligible $\mathcal{O}\left(\frac{k_{L}^{2}}{k_{S}{ }^{2}}\right)$ when late time projection effects are taken into account [57] in the case when the consistency relation is satisfied, but not in our case.

The local quadrupole $Q$ enters in the power spectrum as anisotropic contribution

$$
\left.P_{\mathcal{R}}\left(\vec{k}_{S}\right)\right|_{\gamma\left(\vec{k}_{L}\right)}=P_{\mathcal{R}}\left(\vec{k}_{S}\right)\left[1+\mathcal{Q}_{i j}^{p}\left(\vec{k}_{L}\right) \hat{k}_{S}^{i} \hat{k}_{S}^{j}\right]
$$

and it is defined as the ratio between the consistency-relation-violating contribution of the tensor-scalarscalar bispectrum $\mathcal{B}_{\mathrm{cv}}\left(k_{L}, k_{S}, k_{S}\right)$ and the power spectra of the scalar and tensor modes. Estimating the variance of the quadrupole, that is the observable quantity, it is possibile to extract informations about the parameters of the theory. In our case its value is not so informative in putting constraints on the model with respect to the previous observables.

On the other hand a long wavelength tensor mode can leave "fossil" imprints also on the Large Scale Structure. In this case a tensor mode with wavelength smaller than our observable universe is considered and from an estimator for the tensor power spectrum and its variance it is possibile to extract informations on the minimum size of the galaxy survey on which the tensor can be detected. We report an estimate of the survey size in Appendix C deserving a careful parameter space analysis of the theory in future work. From the estimate we can see that in the next galaxy survey, like EUCLID or even better in 21-cm will be possibile to put bounds and test our theory. So we want to emphasize that even though some (null) searches for power asymmetry in the CMB [3, 64] and Large Scale Structure 65] have already been done, much effort is needed because we have seen how this signatures becomes important in order to rule out inflationary models and also to give informations on the pattern of symmetries in the early universe.

\section{Conclusions}

Using an effective field theory approach to inflation (EFTI), we discussed the case for breaking both time and space diffeomorphism invariance during inflation. While EFTI has been largely applied in the past to study inflationary set-ups with broken time reparameterisation invariance, we pointed out new effects occurring in a set-up where both space and time diffeomorphisms are simultaneously broken during inflation. We provided physical motivations for considering such system, explained how to build an EFT to analyse it. We showed that it can lead to new interesting observational consequences, as a blue spectrum of gravitational waves, enhanced amplitudes for bispectra, and moreover a characteristic angular dependence between the wavevectors in the squeezed limits of bispectra. Although we break space-time diffeomorphisms acting on the coordinates, for simplicity we focus on scenarios that preserve internal rotational and translational symmetries in the space of fields driving inflation. We built actions describing the physics of Goldstone bosons associated with our pattern of symmetry breaking, and the dynamics of vector and tensor modes. In our scenario we find two scalar Goldstone bosons: one scalar $\pi$ associated with the breaking of time reparameterisation, and one scalar $\sigma$ - playing the role of a phonon - associated with the breaking of space translations. Tensor fluctuations enjoy new types of interactions with themselves and with other sectors, associated with operators allowed by the fact that we fully break diffeomorphism invariance.

We discussed observables relative both to scalar and tensor spectra, associated with two and three point functions among fluctuations. We studied both power spectra and bispectra. We determined two broad physical effects that are distinctive of our set-up:

- The first is specific of the scalar sector, and exploits the new couplings between the two scalar Goldstone modes of broken symmetries. We found potentially large contributions to inflationary observables, that can give sizeable effects even in the limit of small breaking of space diffeomorphisms. Such contributions lead to a change in the amplitude of the power spectrum of scalar fluctuations, and, more interestingly, direction dependent contributions (of the form parametrized in [19) to the squeezed limit of the scalar and tensor bispectra. We discussed the physical consequences of these findings, pointing our similarities and differences with previous results in the literature. 
- The second effect is instead more specific of the tensor sector, and exploits novel possibilities for tensors to couple with themselves and with scalars. Such possibilities are associated with operators that are allowed only if we break also space reparameterisation invariance during inflation. They can lead to a blue spectrum for gravitational waves, and moreover to a particular structure for the squeezed limit of tensor-scalar-scalar bispectra, that violate single field consistency relations and can lead to distinctive observable signatures.

Our findings show that the effective field theory for inflation is very well suited for studying situations where all diffeomorphisms are broken during inflation, and suggest new distinctive features for inflationary observables. Future work on this subject can proceed in two different directions. At the observational level, more work is needed to fully characterize the properties of $n$-point functions in these scenarios - possibly not only three but also higher point functions. It would be interesting to find distinctive consistency relations associated with our symmetry breaking pattern, or new observables that specifically test particular features of breaking the space diffeomorphism. At the theoretical level, it would be interesting to find new examples of inflationary models that break all space-time diffeomorphisms (that presumably generalize Solid Inflation) and that then can concretely realize the new observable consequences that we pointed out using a model independent EFT approach. This amounts to characterize physical scenarios - as scalars or higher spin fields - that can break space diffeomorphisms during inflation. Finding explicitly realizations of such set-ups would help also to understand what happens after inflation to whatever physics is responsible for breaking all diffeomorphisms. And possibly, find a mechanism to recover space diffeomorphism invariance, and fully turn off the dynamics of the second Goldstone boson $\sigma$ after inflation ends.

\section{Acknowledgments}

It is a pleasure to thank Matteo Fasiello and Marco Peloso for useful discussions and feedbacks. The work of N.B. was supported in part by ASI/INAF Agreement I/072/09/0 for the Planck LFI Activity of Phase E2. G.T. was supported by an STFC Advanced Fellowship ST/H005498/1.

\section{A Mixing with gravity and decoupling Limit}

In this section we will show why taking the decoupling limit is a consistent approximation in the case under study. Similarly to the equivalence theorem for massive gauge bosons, we expect that the physics of the Goldstone decouples from the transverse modes above a certain energy scale, $E_{m i x}$. For example, in a non-Abelian gauge theory,

$$
\mathcal{L}=-\frac{1}{4} F_{\mu \nu}^{2}-\frac{1}{2}\left(\partial_{\mu} \pi\right)^{2}-\frac{1}{2} m^{2} A_{\mu}^{2}+i m \partial_{\mu} \pi A^{\mu}
$$

where $m^{2}=f_{\pi}^{2} g^{2}$, the decoupling limit is reached taking the limit $g \rightarrow 0, m \rightarrow 0$ with $f_{\pi}$. Therefore, for energies $E>m$, the mixing between the Goldstone and the gauge modes becomes irrelevant and the two sectors effectively decouple.

Just like the gauge theory analogy, in our case we can find a decoupling limit which corresponds to the limits $M_{\mathrm{Pl}} \rightarrow \infty, \dot{H} \rightarrow 0$ with $M_{\mathrm{Pl}}^{2} \dot{H}$ fixed ${ }^{10}$. To see that taking this limit in our case effectively lead to the decoupling of Goldstones and gravity, let us consider first a simplified case, where all the derivatives of $F$ are zero but $F_{X}$. When expanding the the operator $X(9)$ according to (21), one obtains

$$
X=(1+\dot{\pi})^{2} g^{00}+2 \partial_{i} \pi g^{0 i}+\partial_{i} \pi \partial_{j} \pi g^{i j} .
$$

Substituted back into the action (8), the leading mixing of the Goldstone $\pi$ with gravity will be of the form:

$$
\bar{F}_{X} \dot{\pi} \delta g^{00}
$$

\footnotetext{
${ }^{10}$ This is the same as the previous example with the indentifications $g \rightarrow M_{\mathrm{Pl}}^{-1}$ and $m \rightarrow \dot{H}$.
} 
After canonical normalization $\pi_{c} \sim \sqrt{-\bar{F}_{X}} \pi, \hat{g}_{c}^{00} \sim M_{\mathrm{Pl}} \delta g^{00}$ (which gives to the fields the dimension of a mass), we can see that taking the decoupling limit $M_{\mathrm{Pl}} \rightarrow \infty, \dot{H} \rightarrow 0$ with $M_{\mathrm{Pl}}^{2} \dot{H}$ implies that mixing terms becomes irrelevant with respect to the standard kinetic term $\pi_{c}^{2}$ and can be neglected above a certain energy $E_{m i x}$ :

$$
E_{\text {mix }}^{2} \sim \frac{\bar{F}_{X}}{M_{\mathrm{Pl}}^{2}} \sim \frac{\bar{F}_{X}}{M_{\mathrm{Pl}}^{2} H^{2}} H^{2} \sim \epsilon H^{2} \ll H^{2},
$$

where we have used (12) and (17). Therefore as long as $E_{m i x}$ is smaller than $H$, we can safely neglect mixing terms, as they would appear in the action suppressed by powers of $\left(E_{m i x} / H\right)^{2} \sim \epsilon$, since $H$ is our infrared cutoff. The same will happen for the other terms present in the action, but in general the answer depends on which operators are present and significant. For example, from $Z^{i j}$, after the canonical normalization $\sigma_{c} \sim \sqrt{-\bar{F}_{Z}+\bar{F}_{Y^{2}} / 2}$, one has:

$$
\bar{F}_{Z} \dot{\sigma}^{i} g^{0 j} \delta_{i j} \quad \Longrightarrow \quad E_{m i x} \propto \begin{cases}\frac{\alpha \sqrt{-\bar{F}_{Z} / a^{2}}}{M_{\mathrm{Pl}}}, & \text { for } \quad\left|\bar{F}_{Y^{2}}\right| \lesssim\left|\bar{F}_{Z}\right| \\ \frac{-\alpha \bar{F}_{Z} / a^{2}}{M_{\mathrm{Pl}} \sqrt{\bar{F}_{Y^{2}} / a^{2}}}, & \text { for } \quad\left|\bar{F}_{Z}\right| \ll\left|\bar{F}_{Y^{2}}\right|\end{cases}
$$

In the first case, as $\alpha \lesssim 1$,

$$
\frac{E_{m i x}^{2}}{H^{2}} \sim \frac{-\alpha^{2} \bar{F}_{Z} / a^{2}}{M_{\mathrm{Pl}}^{2} H^{2}} \lesssim \epsilon,
$$

where we have used (17). In the second case, $\bar{F}_{Z} \ll \bar{F}_{Y^{2}}$, one can find a similar expression too:

$$
\frac{E_{m i x}^{2}}{H^{2}} \sim \frac{-\alpha^{2} \bar{F}_{Z} / a^{2}}{M_{\mathrm{Pl}}^{2} H^{2}} \frac{\bar{F}_{Z}}{\bar{F}_{Y^{2}}} \lesssim \frac{-\alpha^{2} \bar{F}_{Z} / a^{2}}{M_{\mathrm{Pl}}^{2} H^{2}} \lesssim \epsilon .
$$

Also in this case $E_{m i x}$ is smaller than $H$ and the decopling limit can be safely taken. However, if for example one has $\left|\bar{F}_{Y^{2}}\right| \gg\left|\bar{F}_{Z}\right|$, then, looking at the expansion of the operator $Y_{i} Y^{i}$ one can see that working in the decoupling limit can restrict the range of the allowed parameters:

$$
\bar{F}_{Y^{2}} \dot{\sigma}^{i} g^{0 j} \delta_{i j} \quad \Longrightarrow \quad E_{m i x}^{2} \sim \frac{\alpha^{2} \bar{F}_{Y^{2}} / a^{2}}{M_{\mathrm{Pl}}^{2}}
$$

which is lower than $H$ only if $\bar{Z} \bar{F}_{Y^{2}} / M_{\mathrm{Pl}}^{2} H^{2} \ll 1$.

\section{B Strong coupling}

As it is usual in effective field theories, the non-renormalizable self-interactions of the Goldstone fields will become strongly coupled at a certain energy scale, $\Lambda_{s t}$, beyond which the theory ceases to make sense and new physics must enter. In our case, we have to make sure that $\Lambda_{s t} \gg H$ so that the theory is weakly-coupled in the energy regime we are interested in.

Stronger interactions are related to smaller kinetic energy: indeed if the time-kinetic terms in 22 have prefactors of order $\sim \epsilon$, we would canonically normalize the fields (collectively denoted with $\pi$ for simplicity) like $\epsilon F(\partial \pi)^{2} \sim(\partial \hat{\pi})^{2}$ and inverse power of $\epsilon$ will appear in higher order terms, which would mean stronger interactions or, equivalently, a lower strong coupling scale. Of course, if the coefficients of the kinetic terms were bigger or the coefficients of higher-oder terms were smaller, interactions would be accordingly weaker. As we have to impose a lower bound on $\Lambda_{s t}$, from now on we will focus only on the "worst possible case", when prefactors of time-kinetic terms are as small as $\sim \epsilon F$, while interactions, which are proportional to higher derivatives of $F$ with respect to the operators $X, Y^{i}$ and $Z^{i j}$, are as big as $F$ itself.

Let us first consider the case with speeds of sound very close to unity. In this case, after canonical normalization, we can directly read the strong coupling scale as the scale suppressing higher-order operators in the action, $(\partial \hat{\pi})^{3} / \Lambda^{2}$. The result is simply:

$$
\Lambda_{s t}^{4} \simeq \epsilon^{3} F
$$


If the speed of sound are non-relativistic, the cut-off can not be immediately read from the action as there is an hierarchy between time and spatial derivatives and the theory is not Lorentz invariant. Let's assume for simplicity that $c_{\pi} \simeq c_{\sigma}=c_{s} \ll 1$. We can rescale the time coordinate [67, 68] as $t \rightarrow t / c_{s}$, in order to remove this hierarchy. The quadratic action has now the form:

$$
S_{2} \simeq \int \mathrm{d}^{4} x \sqrt{-g} \epsilon F c_{s}\left(\partial_{\mu} \pi\right)^{2},
$$

and the fields would be normalized as $\epsilon c_{s} F \pi=\hat{\pi}$. Schematically, after canonical normalization, the cubic interactions will have the form

$$
S_{3} \simeq \int \mathrm{d}^{4} x \sqrt{-g} \frac{(\partial \hat{\pi})^{3}}{c_{s}^{5 / 2} \epsilon^{3 / 2} F^{1 / 2}} .
$$

where in the denominator the strong coupling momentum scale appears. We can obtain the energy scale $\Lambda_{s t}$ multiplying by an extra $c_{s}$. The result is:

$$
\Lambda_{s t}^{4} \simeq \epsilon^{3} c_{s}^{9} F
$$

As we said, our theory is under control if $\Lambda_{s t} \gg H$, which will give the constraint:

$$
\epsilon c_{s}^{3} \gg\left(\frac{H}{M_{\mathrm{Pl}}}\right)^{2 / 3}
$$

where we have used the Friedmann equation (12). This is only an order-of-magnitude estimate and, given the many possible combinations of free parameters that are allowed in our action, this constraint can also be not very restrictive. However it is still an important bound to respect for the consistency of the theory.

\section{Tensor fossil estimation}

In order to extract precise informations about the size of the galaxy surveys on which the long wavelength tensor mode can leave "fossil" imprints we need to use the optimal estimator for the tensor power spectrum constructed in [69]. In this case we consider a tensor mode which wavelength is smaller than the size of the observable universe and then we compute the variance of the optimal estimator

$$
\sigma_{\gamma}^{-2}=\frac{1}{2} \sum_{\vec{k}_{L}, p}\left[k_{L}^{3} P_{p}^{n}\left(k_{L}\right)\right]^{-2}
$$

where "p" are the two polarizations of the tensor, $P_{p}^{n}$ is the noise power spectrum, defined as the ratio between the consistency violating contribution to the bispectrum and the total power spectrum

$$
P_{p}^{n}\left(k_{L}\right)=\left[\sum_{\vec{k}_{S}} \frac{\left|\mathcal{B}_{\mathrm{cv}}\left(k_{L}, k_{S},\left|\vec{k}_{L}-\vec{k}_{S}\right|\right) \epsilon_{i j}^{p} \hat{k}_{S}^{i} \hat{k}_{L S}^{j}\right|^{2}\left(k_{L}\right) P^{t o t}\left(k_{S}\right) P^{t o t}\left(\left|\vec{k}_{L}-\vec{k}_{S}\right|\right)}{2}\right]^{-1}
$$

where $V \equiv \frac{(2 \pi)^{3}}{k_{m i n}^{3}}$ is the total volume of the survey and $\epsilon_{i j}^{p}$ is the polarization tensor.

The total power spectrum, that is the measured one, includes both the noise and the signal, $P^{\text {tot }}(k)=$ $P(k)+P^{n}(k)$. The bispectrum can be written in terms of a function $f\left(\vec{k}_{1}, \vec{k}_{2}\right)$, that describes the coupling of the soft mode, and the "long" mode power spectrum $P\left(k_{L}\right)$

$$
B\left(\vec{k}_{L}, \vec{k}_{1}, \vec{k}_{2}\right)=P\left(k_{L}\right) f\left(\vec{k}_{1}, \vec{k}_{2}\right) \epsilon_{i j}^{p}\left(\hat{k}_{L}\right) \hat{k}_{1}^{i} \hat{k}_{2}^{j}=\mathcal{B}\left(k_{L}, k_{1}, k_{2}\right) \epsilon_{i j}^{p}\left(\hat{k}_{L}\right) \hat{k}_{1}^{i} \hat{k}_{2}^{j},
$$

in such a way that the noise power spectrum becomes 69

$$
P_{p}^{n}\left(k_{L}\right)=\left[\sum_{\vec{k}_{S}} \frac{\left|f\left(\vec{k}_{S}, \vec{k}_{L}-\vec{k}_{S}\right) \epsilon_{i j}^{p} k_{S}^{i}\left(k_{L}-k_{S}\right)^{j}\right|^{2}}{2 V P^{t o t}\left(k_{S}\right) P^{t o t}\left(\left|\vec{k}_{L}-\vec{k}_{S}\right|\right)}\right]^{-1} .
$$


$k_{L}$ and $k_{S}$ are the wave number of the long wavelength mode and the short wavelength one. The function $f\left(\vec{k}_{1}, \vec{k}_{2}\right)$ can be easily read from the tensor-scalar-scalar bispectrum (90)

$$
f\left(k_{S}, k_{L}\right)=\frac{C P\left(k_{S}\right)}{k_{S}^{2}} \log \left(\frac{2 c_{\pi} k_{S}}{k_{L}}\right) \quad, \quad C=\frac{\alpha^{2}}{4 c_{\pi}^{2}} \lambda_{1} \lambda_{2},
$$

where we see the novel dependence from the number of modes in the survey. Even if we know that in our case the tensor power spectrum is not exactly scale invariant, at lowest order in $\alpha$ we can assume a nearly scale invariant fiducial power spectrum with amplitude $A_{\gamma}, P_{\gamma}=A_{\gamma} k_{L}^{n_{\gamma}-3}$ with $n_{\gamma} \simeq 0$.

Assuming $P^{(0)}\left(k_{S}\right) / P^{t o t}\left(k_{S}\right) \simeq 1$ ( "correction" to the power spectrum much smaller than 1) if $k_{S} \leq$ $k_{\max }$ and equal to zero otherwise, where $k_{\max }$ is the largest wavenumber that allows for a large signalto-noise measurement, we compute the noise power spectrum. Plugging this quantity in $[106$ and considering that a signal is detected if it has an amplitude larger than $3 \sigma$ we obtain

$$
3 \sigma_{\gamma} \simeq \frac{18 \sqrt{3} \pi^{3 / 2}}{C^{2}}\left(\frac{k_{\min }}{k_{\max }}\right)^{3} \log \left(\frac{2 c_{\pi} k_{\min }}{k_{\max }}\right)^{-2} .
$$

Inverting this relation we can find the size of the galaxy survey necessary to detect at $3 \sigma$ the imprints of primordial tensor mode with a given amplitude $A_{\gamma}$. The estimation, as we can see, would be model dependent and require an improved parameter space analysis of the model, but in order to have a rough estimation, if we assume $c_{\pi} \simeq 10^{-1}$, for the parameter $C$ in the range $(0.1-1)$, one finds that a detectable primordial tensor mode with an amplitude $A_{\gamma} \simeq 2 \times 10^{-9}$, that is a value close to the current upper limits, requires a survey with size in the range $\frac{k_{\max }}{k_{\min }} \sim(4000-1000)$, a value that can be achievable with the next survey like 21-cm [70].

\section{References}

[1] P. A. R. Ade et al. [Planck Collaboration], "Planck 2015 results. XX. Constraints on inflation," arXiv:1502.02114 [astro-ph.CO].

[2] P. A. R. Ade et al. [Planck Collaboration], "Planck 2015 results. XVII. Constraints on primordial non-Gaussianity," arXiv:1502.01592 [astro-ph.CO].

[3] P. A. R. Ade et al. [Planck Collaboration], "Planck 2015 results. XVI. Isotropy and statistics of the CMB," arXiv:1506.07135 [astro-ph.CO].

[4] D. J. Schwarz, C. J. Copi, D. Huterer and G. D. Starkman, "CMB Anomalies after Planck," arXiv:1510.07929 [astro-ph.CO].

[5] J. M. Cornwall, D. N. Levin and G. Tiktopoulos, "Derivation of Gauge Invariance from HighEnergy Unitarity Bounds on the s Matrix," Phys. Rev. D 10 (1974) 1145 [Phys. Rev. D 11 (1975) 972].

[6] C. Cheung, P. Creminelli, A. L. Fitzpatrick, J. Kaplan and L. Senatore, "The Effective Field Theory of Inflation," JHEP 0803 (2008) 014 [arXiv:0709.0293 [hep-th]].

[7] D. Baumann and D. Green, "Equilateral Non-Gaussianity and New Physics on the Horizon," JCAP 1109 (2011) 014 [arXiv:1102.5343 [hep-th]].

[8] S. Endlich, A. Nicolis and J. Wang, "Solid Inflation," JCAP 1310 (2013) 011 [arXiv:1210.0569 [hep-th]].

S. Endlich, B. Horn, A. Nicolis and J. Wang, "Squeezed limit of the solid inflation three-point function," Phys. Rev. D 90 (2014) 6, 063506 [arXiv:1307.8114 [hep-th]].

[9] J. Kang and A. Nicolis, "Platonic solids back in the sky: Icosahedral inflation," arXiv:1509.02942 [hep-th].

[10] A. Gruzinov, Phys. Rev. D 70 (2004) 063518 doi:10.1103/PhysRevD.70.063518 [astro$\mathrm{ph} / 0404548]$. 
[11] L. H. Ford, "Inflation Driven By A Vector Field," Phys. Rev. D 40, 967 (1989);

A. Golovnev, V. Mukhanov and V. Vanchurin, "Vector Inflation," JCAP 0806, 009 (2008) [arXiv:0802.2068 [astro-ph]];

L. Ackerman, S. M. Carroll and M. B. Wise, "Imprints of a Primordial Preferred Direction on the Microwave Background," Phys. Rev. D 75, 083502 (2007) [Erratum-ibid. D 80, 069901 (2009)] [astro-ph/0701357];

M. a. Watanabe, S. Kanno and J. Soda, "Inflationary Universe with Anisotropic Hair," Phys. Rev. Lett. 102, 191302 (2009) [arXiv:0902.2833 [hep-th]];

E. Dimastrogiovanni, N. Bartolo, S. Matarrese and A. Riotto, "Non-Gaussianity and Statistical Anisotropy from Vector Field Populated Inflationary Models," Adv. Astron. 2010, 752670 (2010) doi:10.1155/2010/752670 [arXiv:1001.4049 [astro-ph.CO]];

M. a. Watanabe, S. Kanno and J. Soda, "Imprints of Anisotropic Inflation on the Cosmic Microwave Background," Mon. Not. Roy. Astron. Soc. 412, L83 (2011) [arXiv:1011.3604 [astroph.CO]];

S. Hervik, D. F. Mota and M. Thorsrud, "Inflation with stable anisotropic hair: Is it cosmologically viable?," JHEP 1111, 146 (2011) [arXiv:1109.3456 [gr-qc]];

A. Maleknejad, M. M. Sheikh-Jabbari and J. Soda, "Gauge Fields and Inflation," Phys. Rept. 528, 161 (2013) doi:10.1016/j.physrep.2013.03.003 [arXiv:1212.2921 [hep-th]].

[12] A. Maleknejad, M. M. Sheikh-Jabbari and J. Soda, "Gauge Fields and Inflation," arXiv:1212.2921 [hep-th];

A. Maleknejad and M. M. Sheikh-Jabbari, "Gauge-flation: Inflation From Non-Abelian Gauge Fields," arXiv:1102.1513 [hep-ph];

P. Adshead and M. Wyman, "Chromo-Natural Inflation: Natural inflation on a steep potential with classical non-Abelian gauge fields," Phys. Rev. Lett. 108, 261302 (2012) [arXiv:1202.2366 [hep-th]].

[13] A. E. Gumrukcuoglu, B. Himmetoglu and M. Peloso, "Scalar-Scalar, Scalar-Tensor, and TensorTensor Correlators from Anisotropic Inflation," Phys. Rev. D 81 (2010) 063528 [arXiv:1001.4088 [astro-ph.CO]];

A. E. Gumrukcuoglu, C. R. Contaldi and M. Peloso, "Inflationary perturbations in anisotropic backgrounds and their imprint on the CMB," JCAP 0711 (2007) 005 [arXiv:0707.4179 [astro-ph]];

R. Emami, H. Firouzjahi, S. M. Sadegh Movahed and M. Zarei, "Anisotropic Inflation from Charged Scalar Fields," JCAP 1102, 005 (2011) [arXiv:1010.5495 [astro-ph.CO]];

S. Kanno, J. Soda and M. -a. Watanabe, "Anisotropic Power-law Inflation," JCAP 1012, 024 (2010) [arXiv:1010.5307 [hep-th]];

R. Emami and H. Firouzjahi, "Issues on Generating Primordial Anisotropies at the End of Inflation," JCAP 1201, 022 (2012) [arXiv:1111.1919 [astro-ph.CO]].

K. Yamamoto, M. -a. Watanabe and J. Soda, "Inflation with Multi-Vector-Hair: The Fate of Anisotropy," Class. Quant. Grav. 29, 145008 (2012) [arXiv:1201.5309 [hep-th]];

R. Emami and H. Firouzjahi, "Curvature Perturbations in Anisotropic Inflation with Symmetry Breaking," JCAP 1310, 041 (2013) [arXiv:1301.1219 [hep-th]].

A. A. Abolhasani, R. Emami and H. Firouzjahi, "Primordial Anisotropies in Gauged Hybrid Inflation," arXiv:1311.0493 [hep-th].

X. Chen, R. Emami, H. Firouzjahi and Y. Wang, "The TT, TB, EB and BB correlations in anisotropic inflation," arXiv:1404.4083 [astro-ph.CO].

[14] D. Cannone, G. Tasinato and D. Wands, "Generalised tensor fluctuations and inflation," JCAP 1501 (2015) 01, 029 [arXiv:1409.6568 [astro-ph.CO]].

[15] D. Cannone, J. O. Gong and G. Tasinato, "Breaking discrete symmetries in the effective field theory of inflation," JCAP 1508 (2015) 08, 003 [arXiv:1505.05773 [hep-th]].

[16] L. Graef and R. Brandenberger, "Breaking of Spatial Diffeomorphism Invariance, Inflation and the Spectrum of Cosmological Perturbations," JCAP 1510 (2015) 10, 009 [arXiv:1506.00896 [astroph.CO]]. 
[17] C. Lin and L. Z. Labun, "Effective Field Theory of Broken Spatial Diffeomorphisms," arXiv:1501.07160 [hep-th].

[18] A. A. Abolhasani, M. Akhshik, R. Emami and H. Firouzjahi, "Primordial Statistical Anisotropies: The Effective Field Theory Approach," arXiv:1511.03218 [astro-ph.CO].

[19] M. Shiraishi, E. Komatsu, M. Peloso and N. Barnaby, "Signatures of anisotropic sources in the squeezed-limit bispectrum of the cosmic microwave background," JCAP 1305, 002 (2013) doi:10.1088/1475-7516/2013/05/002 [arXiv:1302.3056 [astro-ph.CO]].

[20] N. Bartolo, S. Matarrese, M. Peloso and A. Ricciardone, "Anisotropic power spectrum and bispectrum in the $f(\phi) F^{2}$ mechanism," Phys. Rev. D 87 (2013) 2, 023504 [arXiv:1210.3257 [astroph.CO]].

[21] N. Bartolo, S. Matarrese, M. Peloso and A. Ricciardone, "Anisotropy in solid inflation," JCAP 1308 (2013) 022 [arXiv:1306.4160 [astro-ph.CO]].

[22] N. Arkani-Hamed and J. Maldacena, "Cosmological Collider Physics," arXiv:1503.08043 [hep-th].

[23] V. A. Rubakov, "Lorentz-violating graviton masses: Getting around ghosts, low strong coupling scale and VDVZ discontinuity," hep-th/0407104.

[24] S. L. Dubovsky, "Phases of massive gravity," JHEP 0410 (2004) 076 [hep-th/0409124].

[25] D. Blas, D. Comelli, F. Nesti and L. Pilo, "Lorentz Breaking Massive Gravity in Curved Space," Phys. Rev. D 80 (2009) 044025 [arXiv:0905.1699 [hep-th]].

[26] L. Senatore and M. Zaldarriaga, "The Effective Field Theory of Multifield Inflation," JHEP 1204 (2012) 024 [arXiv:1009.2093 [hep-th]].

[27] B. A. Bassett, S. Tsujikawa and D. Wands, "Inflation dynamics and reheating," Rev. Mod. Phys. 78 (2006) 537 [astro-ph/0507632].

[28] K. A. Malik and D. Wands, "Cosmological perturbations," Phys. Rept. 475 (2009) 1 doi:10.1016/j.physrep.2009.03.001 [arXiv:0809.4944 [astro-ph]].

[29] M. Shiraishi, D. Nitta, S. Yokoyama and K. Ichiki, "Optimal limits on primordial magnetic fields from CMB temperature bispectrum of passive modes," JCAP 1203, 041 (2012) doi:10.1088/14757516/2012/03/041 [arXiv:1201.0376 [astro-ph.CO]].

[30] M. Shiraishi, "Parity violation of primordial magnetic fields in the CMB bispectrum," JCAP 1206, 015 (2012) doi:10.1088/1475-7516/2012/06/015 [arXiv:1202.2847 [astro-ph.CO]].

[31] N. Bartolo, S. Matarrese, M. Peloso and M. Shiraishi, "Parity-violating and anisotropic correlations in pseudoscalar inflation," JCAP 1501, no. 01, 027 (2015) doi:10.1088/14757516/2015/01/027 [arXiv:1411.2521 [astro-ph.CO]].

[32] N. Bartolo, S. Matarrese, M. Peloso and M. Shiraishi, "Parity-violating CMB correlators with non-decaying statistical anisotropy," JCAP 1507, no. 07, 039 (2015) doi:10.1088/14757516/2015/07/039 [arXiv:1505.02193 [astro-ph.CO]].

[33] J. M. Maldacena, "Non-Gaussian features of primordial fluctuations in single field inflationary models," JHEP 0305 (2003) 013 [astro-ph/0210603].

[34] S. Weinberg, "Quantum contributions to cosmological correlations," Phys. Rev. D 72 (2005) 043514 [hep-th/0506236].

[35] N. Bartolo, M. Peloso, A. Ricciardone and C. Unal, "The expected anisotropy in solid inflation," JCAP 1411 (2014) 11, 009 [arXiv:1407.8053 [astro-ph.CO]].

[36] D. Seery, "Infrared effects in inflationary correlation functions," Class. Quant. Grav. 27 (2010) 124005 [arXiv:1005.1649 [astro-ph.CO]].

[37] M. Gerstenlauer, A. Hebecker and G. Tasinato, "Inflationary Correlation Functions without Infrared Divergences," JCAP 1106 (2011) 021 [arXiv:1102.0560 [astro-ph.CO]].

[38] N. Bartolo, S. Matarrese, M. Pietroni, A. Riotto and D. Seery, "On the Physical Significance of Infra-red Corrections to Inflationary Observables," JCAP 0801, 015 (2008) [arXiv:0711.4263 [astro-ph]]. 
[39] T. Tanaka and Y. Urakawa, "Loops in inflationary correlation functions," Class. Quant. Grav. 30 (2013) 233001 doi:10.1088/0264-9381/30/23/233001 [arXiv:1306.4461 [hep-th]].

[40] X. Chen and Y. Wang, "Quasi-Single Field Inflation and Non-Gaussianities," JCAP 1004 (2010) 027 [arXiv:0911.3380 [hep-th]].

[41] P. Creminelli, M. A. Luty, A. Nicolis and L. Senatore, "Starting the Universe: Stable Violation of the Null Energy Condition and Non-standard Cosmologies," JHEP 0612 (2006) 080 [hepth/0606090].

[42] P. Creminelli and M. Zaldarriaga, "Single field consistency relation for the 3-point function," JCAP 0410 (2004) 006 [astro-ph/0407059].

[43] P. Creminelli, A. Joyce, J. Khoury and M. Simonovic, "Consistency Relations for the Conformal Mechanism," JCAP 1304 (2013) 020 [arXiv:1212.3329].

[44] W. D. Goldberger, L. Hui and A. Nicolis, "One-particle-irreducible consistency relations for cosmological perturbations," Phys. Rev. D 87 (2013) 10, 103520 [arXiv:1303.1193 [hep-th]].

[45] L. Berezhiani and J. Khoury, "Slavnov-Taylor Identities for Primordial Perturbations," JCAP 1402 (2014) 003 [arXiv:1309.4461 [hep-th]].

[46] V. Sreenath and L. Sriramkumar, "Examining the consistency relations describing the three-point functions involving tensors," arXiv:1406.1609 [astro-ph.CO].

[47] A. Kehagias and A. Riotto, "Operator Product Expansion of Inflationary Correlators and Conformal Symmetry of de Sitter," Nucl. Phys. B 864, 492 (2012) [arXiv:1205.1523 [hep-th]].

[48] A. Kehagias and A. Riotto, "Conformal Symmetries of FRW Accelerating Cosmologies," Nucl. Phys. B 884, 547 (2014) [arXiv:1309.3671 [hep-th]].

[49] M. Biagetti, A. Kehagias, E. Morgante, H. Perrier and A. Riotto, "Symmetries of Vector Perturbations during the de Sitter Epoch," JCAP 1307 (2013) 030 [arXiv:1304.7785 [astro-ph.CO]].

[50] P. A. R. Ade et al. [BICEP2 Collaboration], "Detection of $B$-Mode Polarization at Degree Angular Scales by BICEP2," Phys. Rev. Lett. 112 (2014) 24, 241101 [arXiv:1403.3985 [astro-ph.CO]].

[51] P. A. R. Ade et al. [BICEP2 Collaboration], "BICEP2 II: Experiment and Three-Year Data Set," Astrophys. J. 792 (2014) 1, 62 [arXiv:1403.4302 [astro-ph.CO]].

[52] P. A. R. Ade et al. [POLARBEAR Collaboration], "A Measurement of the Cosmic Microwave Background B-Mode Polarization Power Spectrum at Sub-Degree Scales with POLARBEAR," Astrophys. J. 794 (2014) 2, 171 [arXiv:1403.2369 [astro-ph.CO]].

[53] P. A. R. Ade et al. [BICEP2 and Planck Collaborations], Phys. Rev. Lett. 114 (2015) 101301 [arXiv:1502.00612 [astro-ph.CO]].

[54] P. Amaro-Seoane et al. [eLISA] "eLISA/NGO: Astrophysics and cosmology in the gravitationalwave millihertz regime," GW Notes 6 (2013) 4 [arXiv:1201.3621 [astro-ph.CO]].

[55] V. Corbin and N. J. Cornish, "Detecting the cosmic gravitational wave background with the big bang observer," Class. Quant. Grav. 23, 2435 (2006) [gr-qc/0512039].

[56] S. Kawamura et al., "The Japanese space gravitational wave antenna: DECIGO," Class. Quant. Grav. 28, 094011 (2011).

[57] L. Dai, D. Jeong and M. Kamionkowski, "Anisotropic imprint of long-wavelength tensor perturbations on cosmic structure," Phys. Rev. D 88 (2013) 4, 043507 [arXiv:1306.3985 [astro-ph.CO]].

[58] R. Emami and H. Firouzjahi, "Clustering Fossil from Primordial Gravitational Waves in Anisotropic Inflation," JCAP 1510, no. 10, 043 (2015) [arXiv:1506.00958 [astro-ph.CO]].

[59] X. Chen, H. Firouzjahi, M. H. Namjoo and M. Sasaki, "A Single Field Inflation Model with Large Local Non-Gaussianity," Europhys. Lett. 102 (2013) 59001 [arXiv:1301.5699 [hep-th]].

[60] X. Chen, H. Firouzjahi, E. Komatsu, M. H. Namjoo and M. Sasaki, "In-in and $\delta N$ calculations of the bispectrum from non-attractor single-field inflation," JCAP 1312 (2013) 039 [arXiv:1308.5341 [astro-ph.CO]]. 
[61] E. Dimastrogiovanni, M. Fasiello, D. Jeong and M. Kamionkowski, "Inflationary tensor fossils in large-scale structure," JCAP 1412 (2014) 050 [arXiv:1407.8204 [astro-ph.CO]].

[62] J. Kim and E. Komatsu, "Limits on anisotropic inflation from the Planck data," Phys. Rev. D 88 (2013) 101301 [arXiv:1310.1605 [astro-ph.CO]].

[63] E. Dimastrogiovanni, M. Fasiello and M. Kamionkowski, "Imprints of Massive Primordial Fields on Large-Scale Structure," arXiv:1504.05993 [astro-ph.CO].

[64] N. E. Groeneboom and H. K. Eriksen, "Bayesian analysis of sparse anisotropic universe models and application to the 5-yr WMAP data," Astrophys. J. 690, 1807 (2009) [arXiv:0807.2242 [astro-ph]].

[65] A. R. Pullen and C. M. Hirata, "Non-detection of a statistically anisotropic power spectrum in large-scale structure," JCAP 1005, 027 (2010) [arXiv:1003.0673 [astro-ph.CO]].

[66] L. Dai, D. Jeong and M. Kamionkowski, "Seeking Inflation Fossils in the Cosmic Microwave Background," Phys. Rev. D 87 (2013) 10, 103006 [arXiv:1302.1868 [astro-ph.CO]].

[67] L. Senatore and M. Zaldarriaga, "A Naturally Large Four-Point Function in Single Field Inflation," JCAP 1101 (2011) 003 [arXiv:1004.1201 [hep-th]].

[68] S. Endlich, A. Nicolis, R. Rattazzi and J. Wang, "The Quantum mechanics of perfect fluids," JHEP 1104 (2011) 102 [arXiv:1011.6396 [hep-th]].

[69] D. Jeong and M. Kamionkowski, "Clustering Fossils from the Early Universe," Phys. Rev. Lett. 108 (2012) 251301 [arXiv:1203.0302 [astro-ph.CO]].

[70] A. Loeb and M. Zaldarriaga, "Measuring the small - scale power spectrum of cosmic density fluctuations through $21 \mathrm{~cm}$ tomography prior to the epoch of structure formation," Phys. Rev. Lett. 92 (2004) 211301 [astro-ph/0312134]. 